\title{
Mathematical Modelling of Angiogenesis in Wound Healing: Comparison of Theory and Experiment
}

\author{
H. M. BYRNE ${ }^{a+*}$, M. A. J. CHAPLAIN ${ }^{b+\dagger}$, D. L. EVANS ${ }^{c}$ and I. HOPKINSON \\ ${ }^{a}$ School of Mathematical Sciences, University of Nottingham, Nottingham NG7 2RD; ${ }^{\circ}$ Department of Mathematics, University of \\ Dundee, Dundee DDI 4HN: 'Department of Osteoarticular Pathology, University of Manchester, Manchester MI3 $9 P L$
}

(Received July 1999; In final form 4 November 1999)

\begin{abstract}
In this paper we present a simple mathematical model for angiogenesis in wound healing and then compare the results of theoretical predictions from computer simulations with actual experimental data. Numerical simulations of the model equations exhibit many of the characteristic features of wound healing in soft tissue. For example, the steady propagation of the wound healing unit through the wound space, the development of a dense band of capillaries near the leading edge of the unit, and the elevated vessel density associated with newly healed wounds, prior to vascular remodelling, are all discernible from the simulations. The qualitative accuracy of the initial model is assessed by comparing the numerical results with independent clinical measurements that show how the surface area of a range of wounds changes over time. The model is subsequently modified to include the effect of vascular remodelling and its impact on the spatio-temporal structure of the vascular network investigated. Predictions are made concerning the effect that changes in physical parameters have on the healing process and also regarding the manner in which remodelling is initiated.
\end{abstract}

Keywords: Wound healing, angiogenesis, mathematical modelling, experimental data

\section{INTRODUCTION}

Angiogenesis (syn neovascularisation) the formation of new blood vessels, is fundamental for embryonic development and post-embryonic growth, but is rare in adult mammals, except for well-defined cases in the female reproductive tract (Graham \& Lala, 1992) and in tissue-repair processes. Excessive, 'inappropriate' angiogenesis is crucial for tumorigenesis (Folkman, 1976; Folkman \& Brem, 1992; Muthukkaruppan et al., 1982), but is also seen in diabetic retinopathy (Miller et al., 1997; Paques et al., 1997), the rheumatoid pannus (Hamilton, 1983; Myers \& Broom, 1982), atheromatous plaque formation (Paget et al., 1997; Wilcken \& Wilcken, 1997) and following myocardial infarction (Li et al., 1996; Shinohara et al., 1996). The fundamental rôle of angiogenesis in embryonic development, tissue repair responses and tumorigenesis reinforces the well-described similarities between these processes.

Angiogenesis has a central rôle in tissue repair following cutaneous wounding or skeletal fracture. Blood vessels represent up to $60 \%$ of granulation tissue mass and are required to maintain adequate

\footnotetext{
${ }^{*}$ Corresponding Author: E-mail: helen.byrne@ nottingham.ac.uk

†E-mail: chaplain@mcs.dundee.ac.uk
} 
oxygen and glucose levels for the local metabolic demands of healing tissue (Arnold \& West, 1991; Clark, 1988). The extent and rate of angiogenesis may limit repair processes, indicating a potential regulatory rôle for vessel formation. It has been proposed that the major initiator of neovascularisation in repair responses is the relatively hypoxic environment at the centre of wounds, resulting in the generation of an oxygen gradient between this and the relatively well oxygenated edges of such wounds (Knighton et al., 1981). The accumulation of lactic acid and biogenic amines at the wound site and the concentration gradients of these moieties across wounds have also been implicated in the initiation of angiogenesis. These factors may cause injury to mesenchymal cells in the wound, facilitating the release of cytokines and/or growth factors that regulate neovascularisation. An alternative hypothesis proposes that diminished oxygen tension activates cells of the monocyte/macrophage lineage, resulting in the creation of similar cytokines and/or growth factor gradients (Bennet \& Schultz, 1993; Davidson \& Broadley, 1991; Knighton et al., 1983; Knighton \& Fiegel, 1989; Polverini et al., 1977).

The morphological events that are involved in new blood-vessel formation have been defined by studies of in vivo systems such as the chick chorioallantoic membrane and corneal models (Cliff, 1963; Cliff, 1965; Gimbrone et al., 1974) and in vitro examination of endothelial cell migration and proliferation (Sholley et al., 1977; Stokes et al., 1990; Terranova et al., 1985; Ungari et al., 1985). Essentially, new capillaries arise from the sprouting of small venules. Local degradation of parent venule basement-membranes is preceded by endothelial cell phenotype changes. Endothelial cells migrate through the disrupted venular basement membrane towards an angiogenic stimulus and then elongate and align with one another to form a solid sprout. The lumen of the new vessel is formed by the curving of each endothelial cell. Lengthening of the capillary sprouts is facilitated by continued proliferation of endothelial cells in the parent venule. The coalescing of two hollow sprouts results in capillary loop formation and blood flow through the new loop is initiated. New sprouts then develop from the apex of the new loops, propagating angiogenesis (Cliff, 1963; Cliff, 1965: Paweletz \& Knierim, 1989; Schoefl, 1963; Sholley et al., 1984; Warren, 1966). However, as healing progresses neovascularisation is reduced, and mature cutaneous scars are relatively avascular.

The regulation of angiogenesis in tissue repair is complex, involving cytokines and/or growth factors, extracellular matrix components and physiological modulators such as $\mathrm{pH}$ and oxygen tension (Niinikoski et al., 1971). Recently, a multiplicity of angiogenic promoters have been characterised: bFGF; VEGF/VPF; PDECGF; Angiogenin; Angiotropin; EGF; TGF $\beta$ s; and TNF $\alpha$ - these have been reviewed extensively (Davidson, 1995; Phillips et al., 1997; Shah et al., 1995). Fibronectin and heparin have also been shown to potentiate endothelial cell migration and the migration of aortic endothelial cells is dependent upon their synthesizing collagens I, II and III. Fibronectin has been shown to potentiate endothelial cell migration in wounds in vivo and it may be that fibronectin has some rôle in contact guidance of endothelial cell migration in the formation of new capillary beds. The identification of angiogenesis inhibitors active in reducing and remodelling capillary beds during healing has been more difficult, although it may be postulated that increased oxygen tension, resulting from enhanced blood flow in the new capillary networks, and a relative reduction in the metabolic activity of the healing wound are involved in these processes. Several proteins (including thrombospondin, angiostatin and the TGF $\beta$ s) have been shown to inhibit angiogenesis (Canfield and Schor, 1995; Claesson-Welsh et al., 1998; O'Kane and Ferguson, 1998).

The above description of angiogenesis highlights the complexity of just one aspect of wound healing. In recent years a number of authors have developed mathematical models describing various aspects of wound healing and others models of angiogenesis. For example, Sherratt and coworkers have developed models that focus on the migration of fibroblasts and the formation of new tissue during the 
early stages of wound healing (Dale et al., 1994, 1996, 1997; Olsen et al., 1995, 1996, 1997a,b, 1998). More recently, Sherratt and Dallon (1998) have studied the latter stages of wound healing, developing mathematical models that described the reorientation of collagen fibres that occurs during remodelling of scar tissue (Dallon and Sherratt, 1998). Recent work by Anderson and Chaplain
(1998) examines tumour-induced angiogenesis and focusses on the migration of individual endothelial cells at capillary tips.

In this paper we describe a mathematical model for the angiogenesis phase of soft-tissue healing. The key physical variables defined in the model are the capillary-tip density, the positive modulators of angiogenesis and the blood-vessel density. As
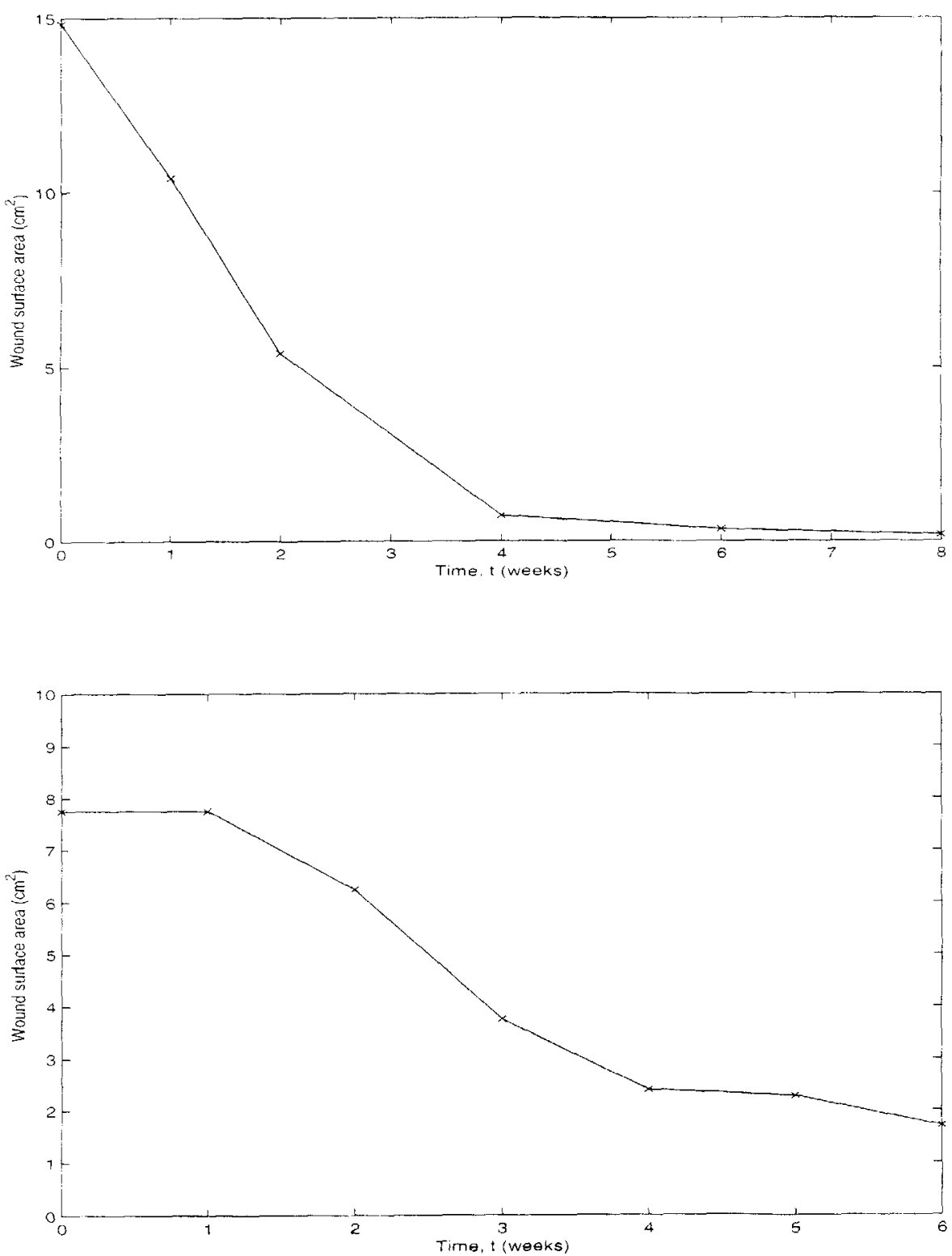

FIGURE 1 Experimental data showing how the surface area $A(t)$ of a wound changes over time when normal healing occurs and also under pathology: (a) normal wound healing; (b) chronic wound healing. See text for details of how the data were collected. 
such, the model is similar in form to existing models of angiogenesis developed in (Chaplain and Byrne, 1996; Pettet et al., 1996a,b) and exhibits many of the characteristic features of angiogenesis. For example, we observe steady propagation of a healing unit through the wound space, together with the development of a dense band of capillaries near the leading edge of the wound healing unit (the brush-border effect). The elevated vessel density associated with newly healed wounds, prior to vascular remodelling, is also observed. However, unlike many previous papers, we then attempt to compare
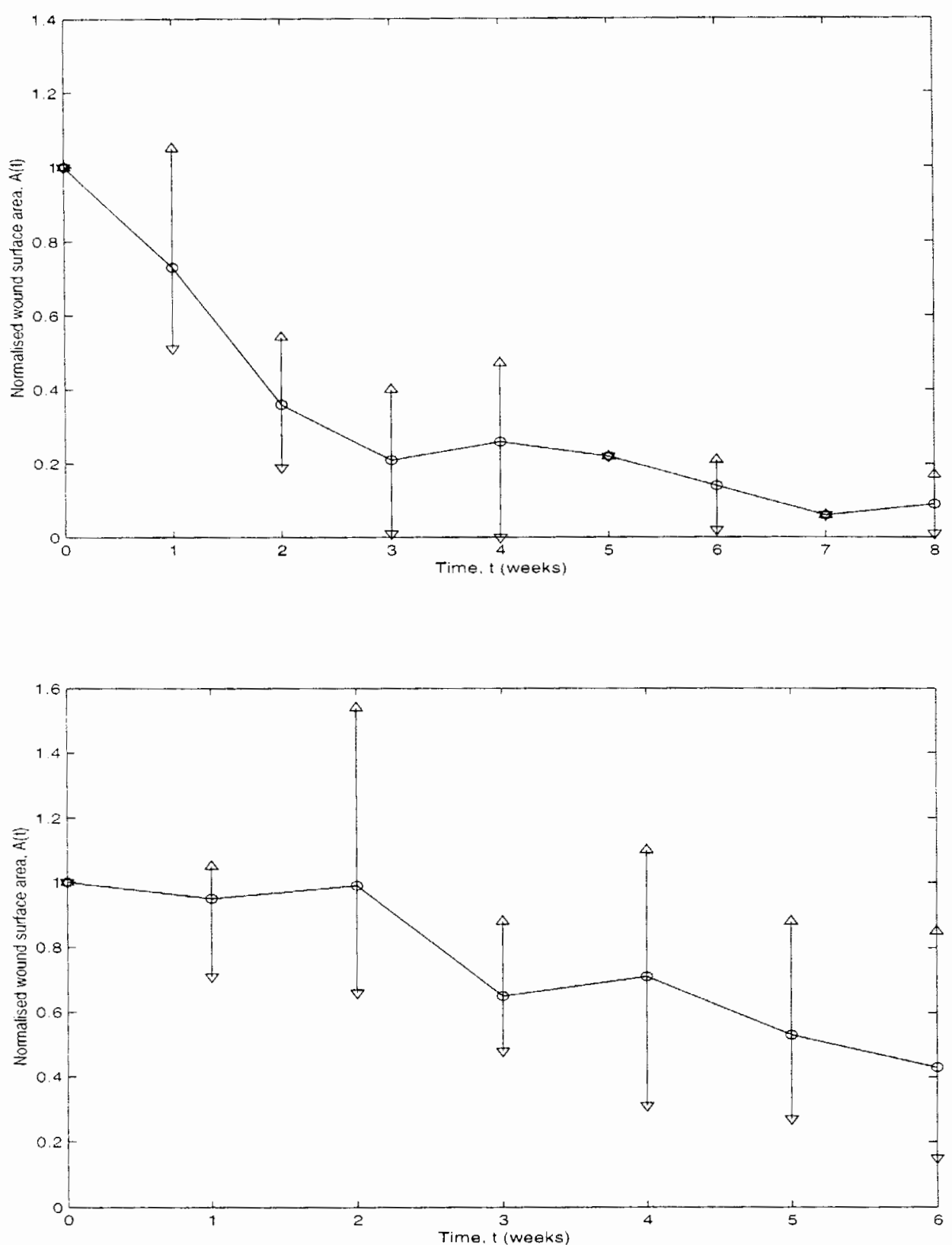

FIGURE 2 Averaged experimental data showing how the surface area $A(t)$ of a wound changes over time when normal healing occurs and also under pathology: (a) normal wound healing; (b) chronic wound healing. 
our results with actual clinical data. An indication of the qualitative accuracy of our mathematical model is provided by comparing the results of our numerical simulations with independent clinical data collected from outpatients attending the Wound Healing Unit at the University of Wales College of Medicine. The healing process was monitored by measuring the surface area of several patients' wounds at regular intervals. As we show, there is good agreement between the theoretical predictions of our model (obtained from numerical simulations) and the clinical results.

We then perform other theoretical experiments by varying certain parameters of the model and examining the effect these changes have on the solutions. The main value to clinicians of these theoretical simulations is the insight that they provide into how changes in the physical parameters affect the healing process and therefore what alternative treatment patients could receive to speed up the healing of their wounds. The simulations may also stimulate the design of in vivo experiments which could be carried out to validate the assumptions and predictions of the mathematical model.

The paper is organised as follows. In Section 2 we present the clinical data collected at the Wound Healing Unit. In Section 3 we develop a mathematical model of angiogenesis whilst in Section 4 numerical simulations obtained from the model are presented. On the basis of these simulations further model modifications are discussed before the numerical simulations are compared with the experimental data. The paper concludes in Section 5 with a summary and discussion of the key results.

\section{THE EXPERIMENTAL RESULTS}

As part of an ongoing programme of research, wounds from 9 out-patients being treated at the Wound Healing Research Unit (University of Wales College of Medicine) were monitored over at period of 10 weeks. During weekly surgeries the dimensions of the wounds were measured and their surface areas calculated. At the end of the 10 week period, the subjects were divided into 2 groups according to whether the wounds had healed normally $(n=4)$ or not $(n=5)$. The criterion used to determine whether a wound had healed was that there had been a $95 \%$ reduction in the surface area during the study. In Figure 1 we present examples from specific patients (one from each group) to show how wound closure varies between patients with normal healing and those suffering from chronic or impaired healing.

To facilitate comparison between the normal and chronic wounds, the data from each wound was rescaled so that the initial surface area was 1.0. The rescaled data from each group was then averaged at each time point and the results are summarised in Tables I and II and graphically in Figure 2. In Tables I and II we present the average area of each wound at each time point (one week intervals) as well as an indication of the largest and smallest wound area in the data set and the number of

TABLE I Summary of data for normal wounds $(n=4)$ showing the average wound area $A_{a n}$ at cach time point (one week intervals). The parameter $N$ denotes the number of measurements used at each specific time point to construct the data

\begin{tabular}{ccccc}
\hline Week & $A_{a v}$ & $A_{\max }$ & $A_{\min }$ & $N$ \\
\hline 0 & 1.00 & 1.00 & 1.00 & 4 \\
1 & 0.73 & 1.05 & 0.51 & 4 \\
2 & 0.36 & 0.54 & 0.19 & 4 \\
3 & 0.21 & 0.40 & 0.01 & 2 \\
4 & 0.26 & 0.47 & 0.00 & 4 \\
5 & 0.22 & 0.22 & 0.22 & 1 \\
6 & 0.14 & 0.21 & 0.02 & 3 \\
7 & 0.06 & 0.06 & 0.06 & 1 \\
8 & 0.09 & 0.17 & 0.01 & 2 \\
\hline
\end{tabular}

TABLE II Summary of data for chronic wounds $(n=5)$ showing the average wound area $A_{a}$ at each time point (one week intervals). The parameter $N$ denotes the number of measurements used at each specific time point to construct the data

\begin{tabular}{ccccc}
\hline Week & $A_{a n}$ & $A_{\max }$ & $A_{\min }$ & $N$ \\
\hline 0 & 1.00 & 1.00 & 1.00 & 5 \\
1 & 0.95 & 1.05 & 0.71 & 5 \\
2 & 0.99 & 1.54 & 0.66 & 5 \\
3 & 0.65 & 0.88 & 0.48 & 5 \\
4 & 0.71 & 1.10 & 0.31 & 5 \\
5 & 0.53 & 0.88 & 0.27 & 5 \\
6 & 0.43 & 0.85 & 0.15 & 5 \\
\hline
\end{tabular}


measurements used in calculating the averages $(N)$. From the data we note that the rate of closure in the normal wounds is initially rapid and slows down as the wound closes. Also, the surface area decreases monotonically over time. By contrast, the chronic wounds are characterised by an initial increase in the wound surface area, followed by closure of the wound at a considerably slower rate than for the normal wounds. We note also that, perhaps because our study is not large, there is considerable variation in the way in which both normal and chronic wounds heal.

\section{THE MATHEMATICAL MODEL}

We now present our mathematical model of wound healing angiogenesis. Attention focusses on three physical variables: the capillary tip density $n$, the concentration of positive modulators of angiogenesis i.e. angiogenic factors (AF) $a$, and the blood vessel density $b$. We remark that no distinction is made between primary and secondary vessels, or between the large number of AFs known to be present in the wound space, these assumptions being made to minimise the complexity of the model. Further, we neglect the effect of inhibitors of angiogenesis that may be present in the wound space. By focussing on epidermal wounds whose initial width $O(\mathrm{~cm})$ is considerably larger than the thickness of the epidermis $O\left(10^{-2} \mathrm{~cm}\right)$, it is possible to regard wound healing as a two-dimensional process which occurs essentially in the same plane as the skin itself (that is, in a plane perpendicular to the depth of the epidermis). If we assume further that the wound remains approximately radially symmetric as it closes then it is possible to restrict attention to just one spatial dimension, that of the wound radius $r$. Thus our three dependent variables, $n, a, b$, are functions of time $t$ and the spatial variable $0<r<R$, with the $r$ direction parallel to the direction of tip growth, and such that the wound centre is located at $r=0$ and the initial wound margin lies at $0<r=R_{0}$. The description in the introduction of tip sprouting, migration and anastomosis motivates our separate treatment of the tip density and the blood vessel density. In order to minimise the complexity of the resulting model, in addition to the simplifications outlined above, no explicit mention is made of either the oxygen concentration or the fibroblast density in the wound space (a more detailed model which includes these variables may be found in Pettet et al., 1996). In summary then, our model consists of conservation equations for $n, a$ and $b$. In words, these equations express the fact that for a given species, $X$ say, the following balance prevails:

$$
\begin{aligned}
\left(\begin{array}{c}
\text { rate of accumula- } \\
\text { tion of species } X
\end{array}\right)= & \left(\begin{array}{c}
\text { net flux of } X \\
\text { through boundaries } \\
\text { of control volume }
\end{array}\right) \\
& +\left(\begin{array}{c}
\text { net production rate } \\
\text { of } X \text { within volume }
\end{array}\right)
\end{aligned}
$$

The precise equations are presented below with a description of each term in the model in words above. The equations are similar in form to existing models of angiogenesis upon which the present study is based (Byrne and Chaplain, 1995; Chaplain and Byrne, 1996; Pettet et al, 1996a,b). We now explain the various terms in each of the equations.

\section{Capillary Tip Density Equation}

The dominant factors affecting the capillary tip density $n$ are assumed to be tip migration, proliferation, anastomosis and natural decay. Based on Sherratt and Murray's findings (1990) that the random motility coefficient for epithelial cells may be three orders of magnitude smaller than the diffusion coefficient for chemicals, we include a small contribution to tip migration due to random motility, and assume that the AF provides the dominant mechanism for tip migration, directing tip motion up spatial gradients of $a$, into the wound space. Such motion is termed chemotaxis. Tip proliferation arises from secondary branching, with tips emanating from pre-existing blood vessels, and stimulated by the presence of the AF. Thus we postulate that tip proliferation, or budding, is proportional to both $b$ and $a$, with constant of proportionality $\lambda_{0}$ (equally we could include a saturation term in the tip proliferation term of the form $\lambda_{0} a b(\hat{B}-b)$ where $\hat{B}$ is a threshold vessel density: this would limit tip 
proliferation to a finite range of vessel densities, $b \in(0, \hat{B}))$. For simplicity we assume that natural decay and tip-to-tip anastomosis constitute the dominant contributions to tip loss, and neglect other loss mechanisms, such as tip-to-branch anastomosis. Natural death is modelled by a linear decay term, with rate constant $\lambda_{1}$. To reflect the fact that (at least) two tips must fuse to effect tip-to-tip anastomosis, we introduce an additional loss term proportional to $n^{2}$, with rate constant $\lambda_{2}$. Combining these effects and denoting by $\mu_{n}$ and $\chi$ the assumed constant random motility and chemotaxis coefficients respectively, we express the rate of change of tip density through the following equation:

$$
\begin{aligned}
\left(\begin{array}{c}
\text { rate of increase } \\
\text { of tip density }
\end{array}\right)= & \left(\begin{array}{c}
\text { rate of tip migration } \\
\text { due to random motility } \\
\text { + chemotaxis }
\end{array}\right) \\
& +\left(\begin{array}{c}
\text { rate of } \\
\text { budding }
\end{array}\right)-\left(\begin{array}{c}
\text { rate of } \\
\text { natural } \\
\text { decay }
\end{array}\right) \\
& -\left(\begin{array}{c}
\text { rate of } \\
\text { anastomosis }
\end{array}\right)
\end{aligned}
$$

or, equivalently,

$$
\begin{aligned}
\frac{\partial n}{\partial t}= & \frac{\mu_{n}}{r} \frac{\partial}{\partial r}\left(r n \frac{\partial n}{\partial r}\right)-\frac{\chi}{r} \frac{\partial}{\partial r}\left(r n \frac{\partial a}{\partial r}\right) \\
& +\lambda_{0} a b-\lambda_{1} n-\lambda_{2} n^{2} .
\end{aligned}
$$

\section{Angiogenic Factor (AF) Concentration Equation}

We assume that the AF spreads throughout the wound via diffusion with a constant diffusion coefficient. To approximate the mitigating effect that the local (blood-borne) oxygen has on AF production by either the injured mesenchymal cells or macrophages present in the wound space, we assume that $\mathrm{AF}$ production is dependent upon the degree of disruption to the vasculature and hence to the local blood vessel density. In particular, we assume that if the local vessel density is below some characteristic value, $b_{\text {char }}$ say, then attractant production occurs at the constant rate $\lambda_{3}$; if $b>b_{\text {char }}$ then the production rate is negligible; as the vessel density decreases through $b_{\text {char }}$ the attractant production rate increases smoothly from zero to $\lambda_{3}$. We assume that natural decay and removal via the local vascular network are the dominant mechanisms for $\mathrm{AF}$ loss, these processes occurring at rates $\lambda_{4}$ and $\lambda_{5}$ respectively. Using $D$ to denote the assumed constant diffusion coefficient, and combining the above effects, we express the rate of change of the $\mathrm{AF}$ concentration through the following equation:

$$
\begin{aligned}
\left(\begin{array}{c}
\text { rate of } \\
\text { increase of } \mathrm{AF} \\
\text { concentration }
\end{array}\right)= & \left(\begin{array}{c}
\text { diffusion } \\
\text { of } \mathrm{AF}
\end{array}\right)+\left(\begin{array}{c}
\text { rate of } \\
\mathrm{AF} \\
\text { production }
\end{array}\right) \\
& -\left(\begin{array}{c}
\text { rate of } \\
\text { decay } \\
\text { of } \mathrm{AF}
\end{array}\right)-\left(\begin{array}{c}
\text { rate of } \\
\text { removal } \\
\text { via blood } \\
\text { vessels }
\end{array}\right)
\end{aligned}
$$

or, equivalently,

$$
\begin{aligned}
\frac{\partial a}{\partial t}= & \frac{D}{r} \frac{\partial}{\partial r}\left(r \frac{\partial a}{\partial r}\right) \\
& +\frac{\lambda_{3}}{2}\left(\tanh \left(\frac{b_{\text {char }}-b}{\Delta b}\right)+1\right) \\
& -\lambda_{4} a-\lambda_{5} a b .
\end{aligned}
$$

\section{Blood Vessel Density Equation}

We assume that vessel production occurs as a consequence of tip migration, with new cells being deposited behind the tip at a rate which maintains contiguity of the vessel. Branch loss may be incorporated to describe the remodelling process by which the elevated level of vasculature associated with newly repaired wounds is reduced to a level close to that of undamaged tissue. This process occurs by shortening and thickening of the blood vessels and typically takes place over a longer period ( $\sim$ months) than the migration of the healing unit ( $\sim 2$ weeks). For this reason, it is not considered in this initial model. However the effect of remodelling will be considered in a subsequent section. Thus, the equation describing the evolution of the vessel density which we propose is given by:

$$
\left(\begin{array}{c}
\text { rate of increase } \\
\text { of vessel density }
\end{array}\right)=\left(\begin{array}{c}
\text { rate of } \\
\text { deposition by tips }
\end{array}\right)
$$


or, equivalently,

$$
\frac{\partial b}{\partial t}=\mu_{n} n \frac{\partial n}{\partial r}-\chi n \frac{\partial a}{\partial r} .
$$

\section{Boundary and Initial Conditions}

In order to close Equations (1-3) initial and boundary conditions are imposed. We assume that initially the wound is devoid of tips, vessels and AF and that the wound margin lies at $r=R_{0}$ (see Equations (4) and (5)). In addition, we assume that the wound is symmetric about its centre $r=0$ (see Equation (6)). The tip density at the wound margin is also prescribed (see Equations (4) and (7)). In particular we assume that there are some tips present at the wound margin at $t=0$, such tips being connected to the normal tissue that surrounds the wound. In order to mimic the surge in endothelial cell density that is an early response to wounding, we assume that the tip density at the wound margin decays exponentially. Finally, blood-borne removal of $\mathrm{AF}$ at the wound margin is modelled by assuming that the flux of $\mathrm{AF}$ out of the wound is proportional to both $a(R, t)$ and $b(R, t)$. Introducing the parameters $N_{0}, k_{1}$ and $k_{2}$ to represent respectively the magnitude of the surge in tip density during early wounding, the rate at which the surge in capillary tips decay away and the rate at which the AF is removed from the wound region, the above conditions can be formulated mathematically as follows:

$$
\begin{aligned}
& n(r, 0)=\left\{\begin{array}{ll}
0 & \text { for } r \in(0, R) \\
N_{0} & \text { for } r=R,
\end{array} a(r, 0)=0,\right. \\
& b(r, 0)= \begin{cases}0 & \text { for } r \in\left(0, R_{0}\right) \\
b_{n o r m} & \text { for } r \in\left(R_{0}, R\right),\end{cases} \\
& \frac{\partial n}{\partial r}(0, t)=\frac{\partial a}{\partial r}(0, t)=0, \\
& n(R, t)=N_{0} e^{-k_{1} t}, \\
& D \frac{\partial a}{\partial r}(R, t)=-k_{2} a(R, t) b(R, t) \quad \text { for } t>0 \text {. }
\end{aligned}
$$

We remark that whilst the boundary and initial conditions above are well-posed and consistent, there are discontinuities in the initial tip and vessel densities. However, given the physical situation being modelled, these discontinuities are natural, reflecting the underlying biology: the wound margin is a sharp interface between the ordinary healthy and the wounded tissues. We note finally that when other choices of the initial conditions, in which these discontinuities were smoothed out, were employed, the numerical results (not presented) were qualitatively similar to those presented in the next section (and obtained with the discontinuous initial conditions stated above). Thus we infer that the limiting behaviour of our system is, in some sense, stable and insensitive to a range of biologically reasonable initial conditions.

\section{RESULTS}

\subsection{Numerical Simulations}

Before being used to construct numerical solutions, the model equations were reformulated in terms of dimensionless variables, for example $r$ was scaled with the initial wound radius $R_{0}(\sim 2 \mathrm{~cm}), t$ with a typical timescale for neovascularisation ( $\sim 2$ weeks) and $b$ with a characteristic vessel density (see appendix for details). When carrying out the rescaling, where possible, parameter values were calculated from experimental data. In the absence of such data, values estimated and used in other models were employed (Stokes \& Lauffenburger, 1991: Balding $\&$ McElwain, 1985). The advantage of recasting the model equations in terms of dimensionless variables is that it enables the relative roles of the different physical processes present in the model to be assessed and may be used to justify the neglection of mechanisms not present in the model, such as random motion of the blood vessels.

The dimensionless form of Equations ( $1-7$ ) were solved numerically using a package from the NAG library (routine DO3PCF). This method uses finite difference approximations to perform a spatial discretisation of the model equations, thereby reducing them to a system of ordinary differential equations which are integrated using the method of lines.

The simulations presented in Figure 3 show how the key physical variables evolve during normal healing. In the absence of a functioning vasculature, the AF is rapidly produced in the centre of the 

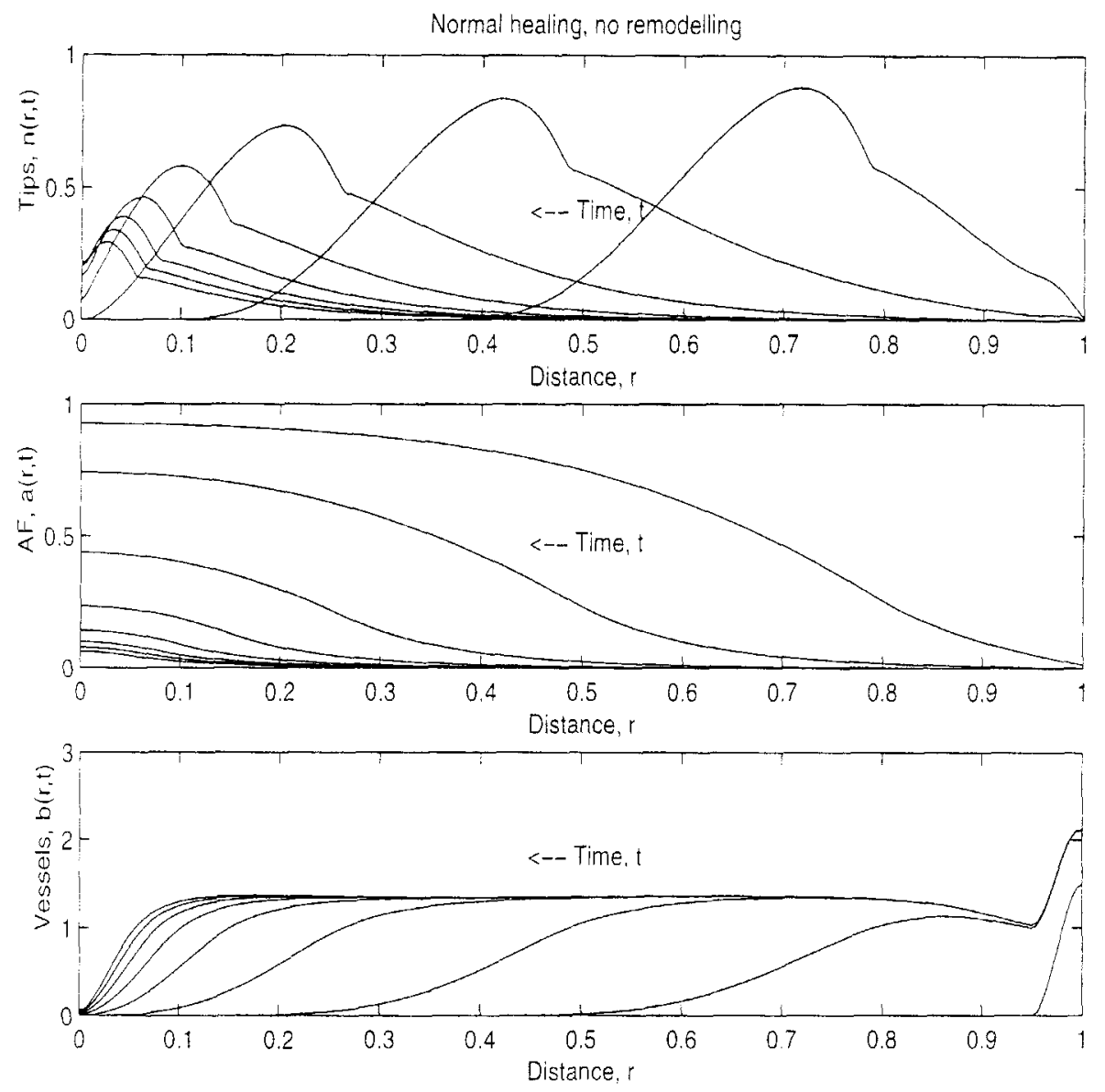

FIGURE 3 Here we present the results of numerical simulations for which successful healing occurs for two different values of $\chi$. The centre of the wound is located at $r=0$ with the edge at $r=1$. The direction of motion is from right to left. The three dependent variables are plotted at times $t=0.5,1.0,1.5, \ldots, 4.0$ : For case (a) we have $\chi=0.4$ and we plot: (i) profiles of the capillary tip density $(n)$; (ii) profiles of the $\mathrm{AF}$ concentration $(a)$ : (iii) profiles of the blood vessel density $(b)$. Parameter values: $\mu_{n}=0.001, x=0.4$. $D=1, \lambda_{0}=50, \lambda_{1}=10, \lambda_{2}=10, \lambda_{3}=25=\lambda_{4} . \lambda_{5}=25, R=1, R_{0}=0.95, k_{1}=2, N_{0}=1=b_{\text {norm }}=b_{\text {char }}, \Delta b=0.0 \mathrm{l}$. For case (b) we reduce $\chi$ from 0.4 to 0.1 , holding all other parameter values fixed. We plot profiles of the capillary tip density and the vessel density at times $t=0.5,1.0,1.5, \ldots 4.0$ (solid lines) and $t=50,60,70 \ldots .100$ (dotted lines). Parameter values: as per Figure 3(a). except that $\chi=0.1$.

wound. Blood-borne removal of the AF near the wound margin leads to the formation of a spatial gradient in AF there. New capillary tips are formed predominantly at the interface between the wound margin and the AF. The capillary tips migrate via chemotaxis up the spatial gradient in the AF towards the wound centre, leaving a trail of blood vessels in their wake. In this way the developing vasculature migrates towards the wound centre. As the vessel density in the tissue increases the rate of $\mathrm{AF}$ production falls and the number of capillary tips being formed diminishes. The AF concentration gradually falls to zero and the healing process slows down, eventually stopping when the wound closes. The results presented in Figure 3 suggest that the speed with which the healing unit migrates into the wound changes over time: the healing unit travels fastest during the initial stages and slows down during the latter stages of healing. This is in good agreement with independent experimental observations (Muthukarruppan et al., 1982). The profiles of the vessel density show that it is higher than the corresponding, normal level in the healed tissue. This is a common feature of newly healed wounds. In 

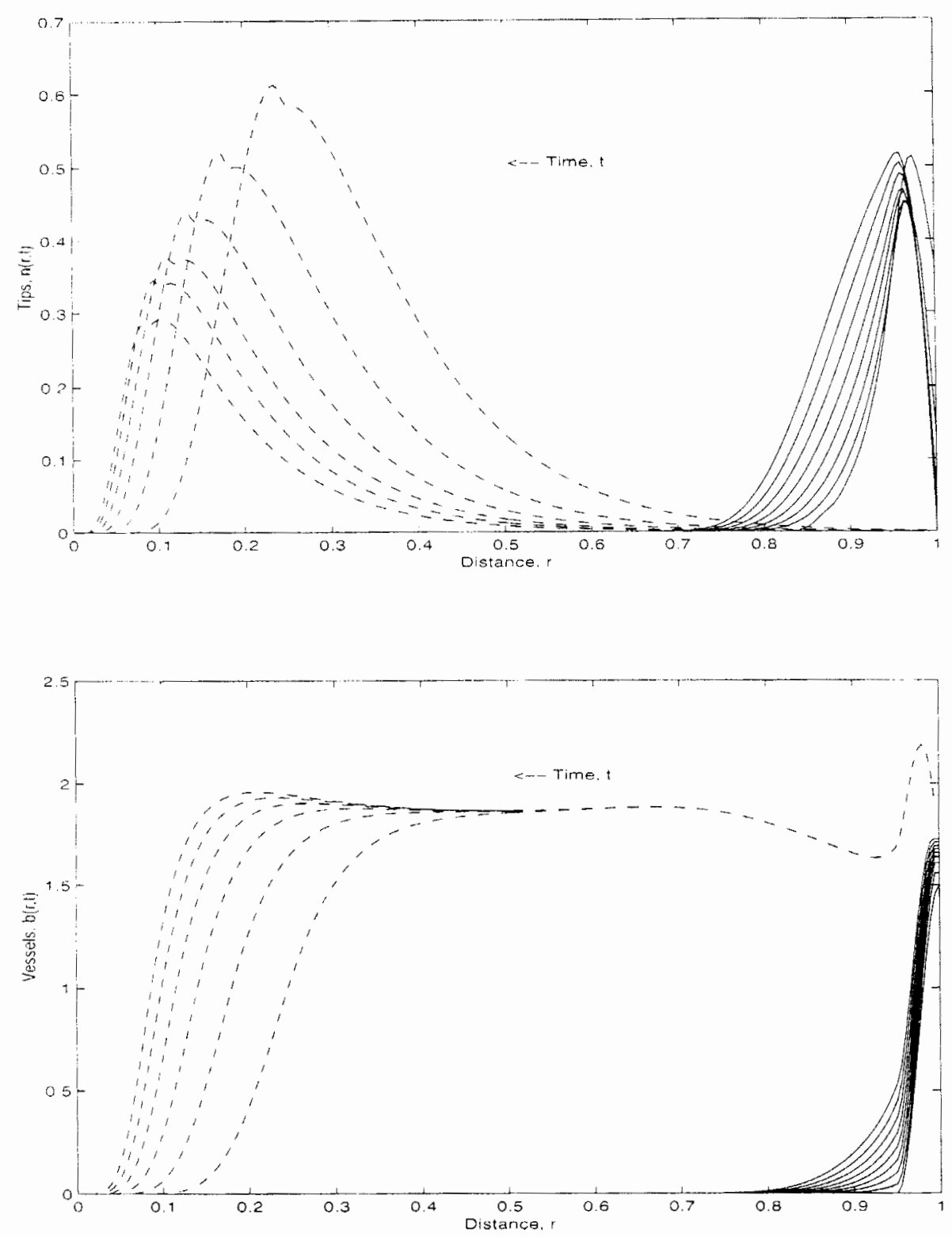

FIGURE 3 (Continued).

practice a remodelling term should be included in the model equations to restore the vessel density to a normal level: its incorporation is discussed below.

The simulations presented in Figure 3(b) show how the healing process is affected when the chemotaxis coefficient $\chi$ decreases (from $\chi=0.4$ in Figure 3(a) to $\chi=0.1$ ). Over the same time period as the simulations in Figure 3(a) (up to $t=4$ ), there is a marked reduction in both the penetration depth and the density of the invading capillary tips. However, healing of the wound does occur over a longer timescale in a qualitatively similar manner. These results are of relevance when comparing our numerical simulations with the actual wound healing data (see Figure 9). Also, a full sensitivity analysis of the effect of changing the parameter $\chi$ is presented later in this section and results are given in Figure 10. 
The simulations presented in Figure 4 show how the healing process is affected when the rate of tip production falls (from $\lambda_{0}=50$ in Figure 3 to $\lambda_{0}=5$ ). In Figure 4(a) (which is the analogue of Figure $3(\mathrm{a})$ ) tip production and invasion are minimal, the levels of AF production remain high and, whilst there is a small amount of vessel outgrowth, the vessel density remains low. Consequently it takes considerably longer for the wound to close than in Figure 3. In Figure 4(b) we present results showing how the wound healing process is affected when $\chi$ is reduced to a value of 0.1 . Similar remarks regarding the time taken for closure can be made as for the results of Figure 3(b) above. Once again these simulations have direct relevance when comparing the numerical results with the actual wound healing data later in Figure 9.

Guided by these simulations, we predict that a possible mechanism for poor wound healing in, for example, diabetic patients may be an impaired response of the endothelial cells that constitute the blood vessels to growth factors which are present in the wound space and which normally stimulate endothelial cell proliferation.

Guided by the results presented in Figures 3 and 4 we infer that reductions in either the rate of capillary tip production $\lambda_{0}$ or the chemotaxis coefficient $\chi$ impair healing. However the two mechanisms produce slightly different effects: when $\chi$ is reduced penetration and proliferation of the capillary tips are
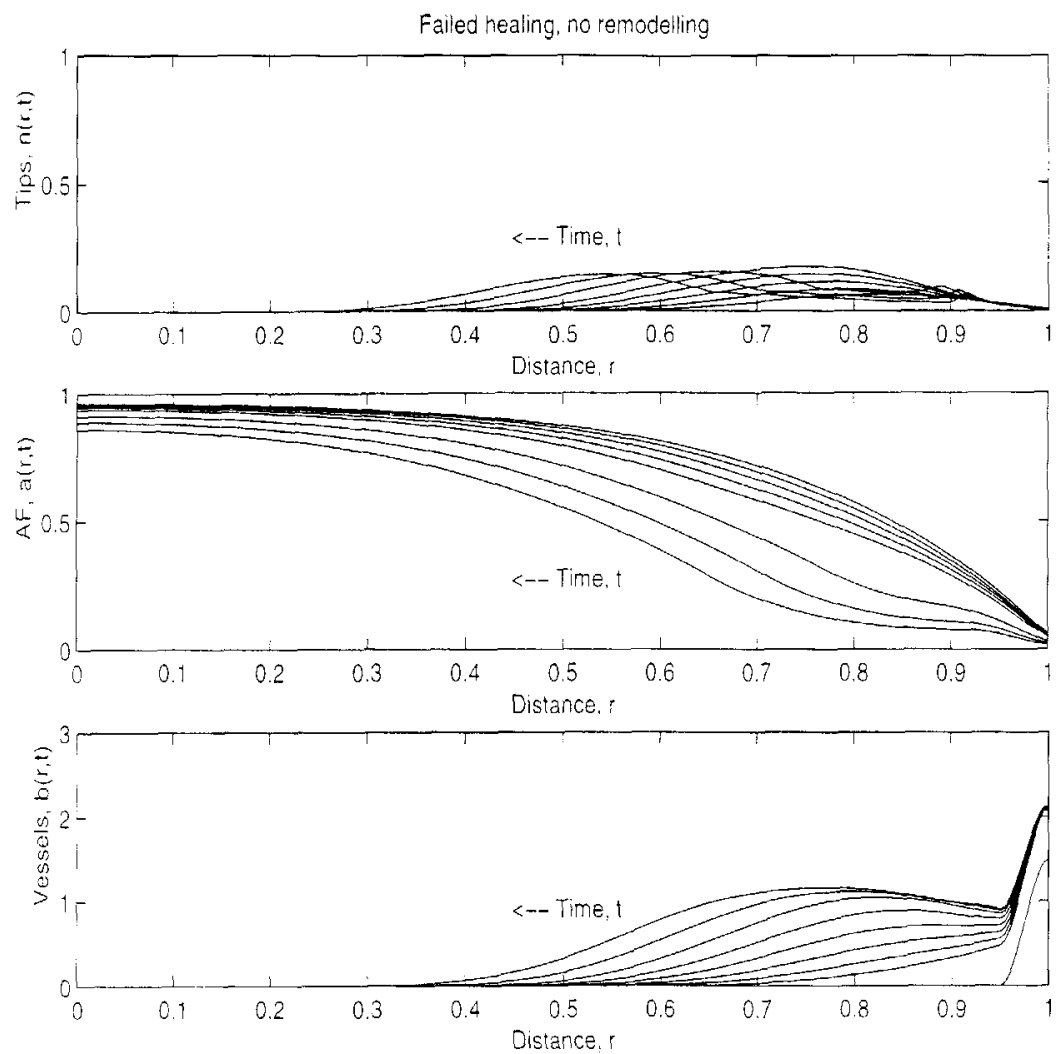

FIGURE 4 Here we present numerical results which show how the healing process is affected when the rate of tip formation is reduced from $\lambda_{0}=50$ (Figure 1) to $\lambda_{0}=5$. As the simulations show. in this case healing fails. Motion is again from right to left. In case (a) the three dependent variables are plotted at times $t=0.5,1.0,1.5, \ldots, 4.0$ : (i) profiles of the capillary tip density $(n)$; (ii) profiles of the $\mathrm{AF}$ concentration $(a)$; (iii) profiles of the blood vessel density $(b)$. Parameter values: $\mu_{n}=0.001 . \chi=0.4, D=1$. $\lambda_{0}=5, \lambda_{1}=10, \lambda_{2}=10, \lambda_{3}=25=\lambda_{4}, \lambda_{5}=25, R=1 . R_{0}=0.95, k=2, N_{0}=1=b_{\text {nom }}=b_{\text {char }}, \Delta b=0.01$. For case (b) we reduce $\chi$ from 0.4 to 0.1 , holding all other parameter values fixed. We plot profiles of the capillary tip density and the vessel density at times $t=0.5,1.0,1.5, \ldots .4 .0$ (solid lines) and $t=50,60,70 \ldots 100$ (dotted lines). Parameter values: as per Figure $4($ a), except that $\chi=0.1$. 

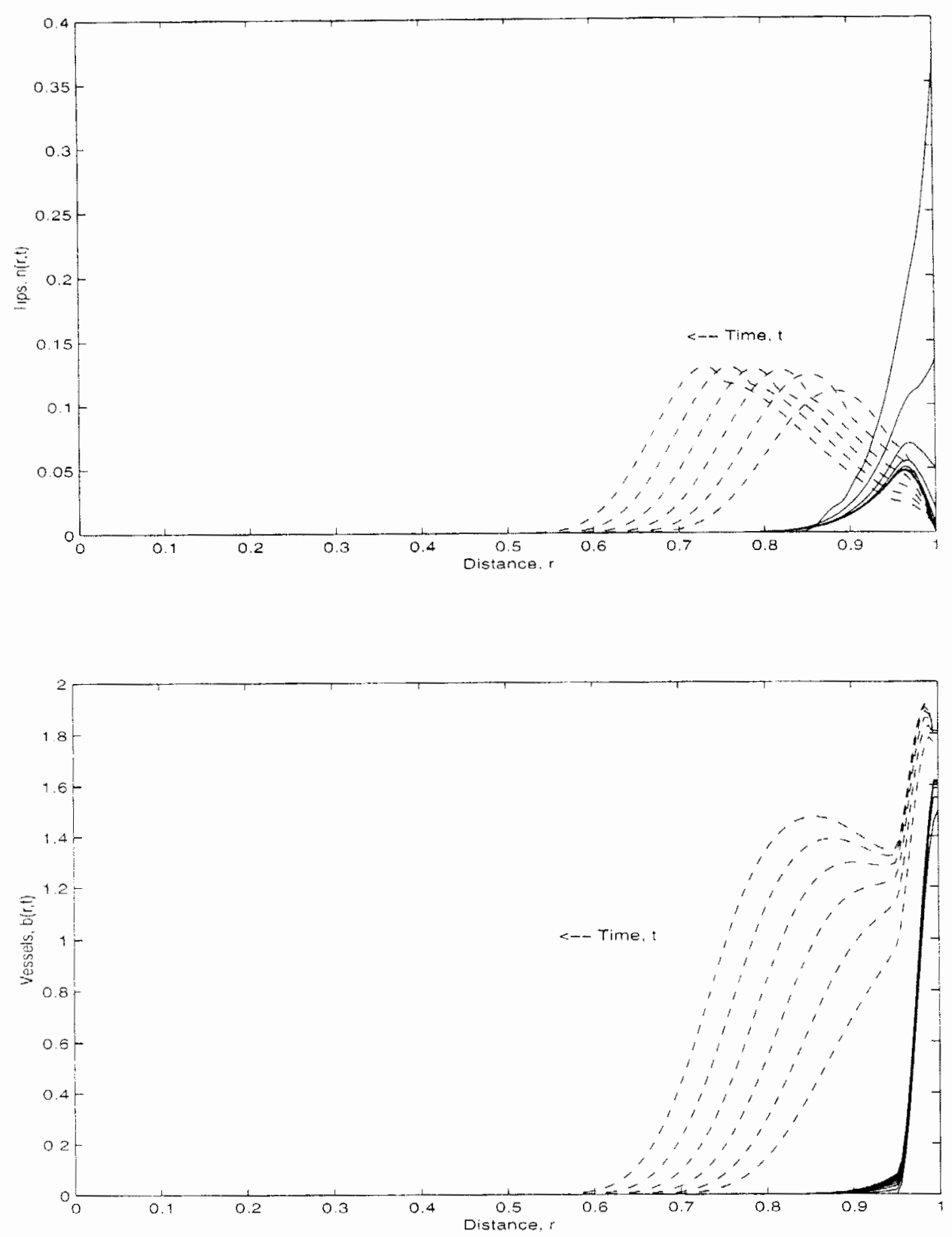

FIGURE 4 (Continued).

both diminished whereas when $\lambda_{0}$ is reduced only the tip density seems to diminish and the reduction in capillary tip penetration is less dramatic. By exploiting these results it may be possible to predict the cause of impaired wound healing: reasonable penetration of low capillary tip density may correspond to a reduction in the tip proliferation rate whereas poor penetration and low capillary tip density may be ascribed to a reduction in the sensitivity of the cells to the chemotrattactants being produced in the wound space.

In Section 2 we presented clinical measurements showing how the surface area of normal and chronic wounds changes over time. In order to facilitate 
comparison with this data it is necessary to extract from the numerical simulations an estimate of the wound's surface area. We do this by tracking the position of the leading front of the migrating capillary tips $r=R(t)$ and assuming that the surface area of the wound is equal to its avascular surface area $A(t)=\pi R^{2}$. Normalised plots of $A(t) / A(0)$ for the simulations of Figures 3 and 4 are presented in Figure 5. The qualitative behaviour in each case is the same: $A(t)$ decreases in a bi-exponential manner with an initially rapid decrease in $A(t)$ followed by a slower decay to the healed state. Such behaviour is widely observed in healing wounds and is consistent with the experimental results presented in Section 2. We remark that initially $(0<t<1)$, from the sketch of $A(t)$, our model is unable to distinguish between the normal and chronic wounds since they both decay at the same rapid rate. However for $t>1$ the difference between the two wounds is apparent, with $A(t)$ decaying to zero more rapidly for the normal wound than for the chronic wound.

As stated above, another key feature of newly healed wounds is overhealing. A simple measure of the degree of overhealing within a wound can be obtained by computing the mean vessel density $B(t)=2 \int_{0}^{1} b(r, t) r d r$ (so that $B(t)=1$ corresponds to normal tissue and $B(t)>1$ to newly healed wounds). In Figure 6 we show how $B(t)$ evolves for the numerical simulations presented in Figures 3 and 4. In the normal wound, we observe that $B(t) \rightarrow 1.3$

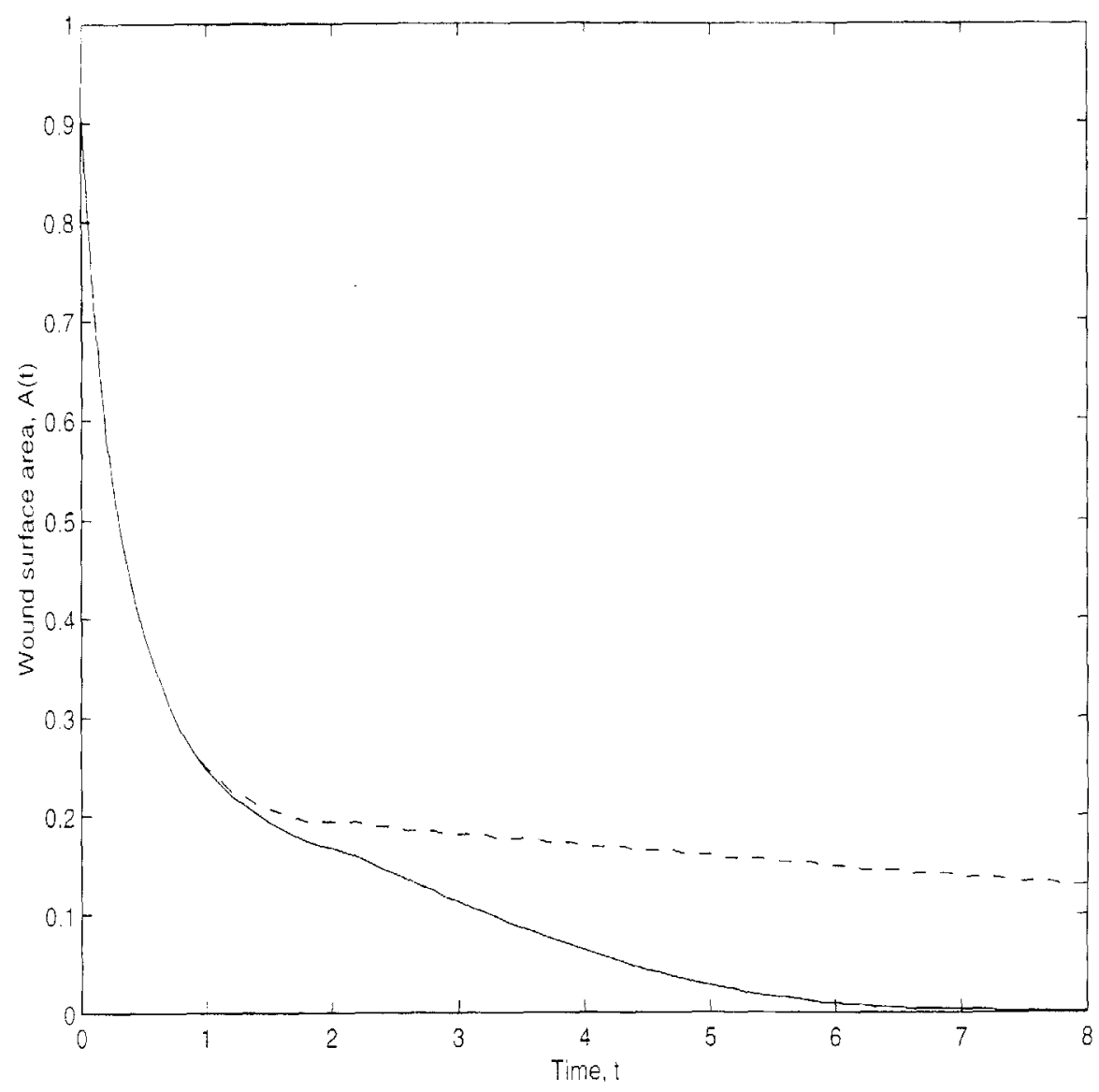

FIGURE 5 Here we present numerical profiles that show how the wound surface area $A(t) / A(0)$ changes over time for the numerical simulations presented in Figures 3 and 4 . The surface area $A(t)$ is determined by tracking the position of the leading edge of the vascular front $R(t)$ and assuming that $A(t)=\pi R^{2}(t)$. Key: successful healing (solid line): failed healing (dashed linc). Parameter values: as per Figures $3(\mathrm{a})$ and $4(\mathrm{a})$. 


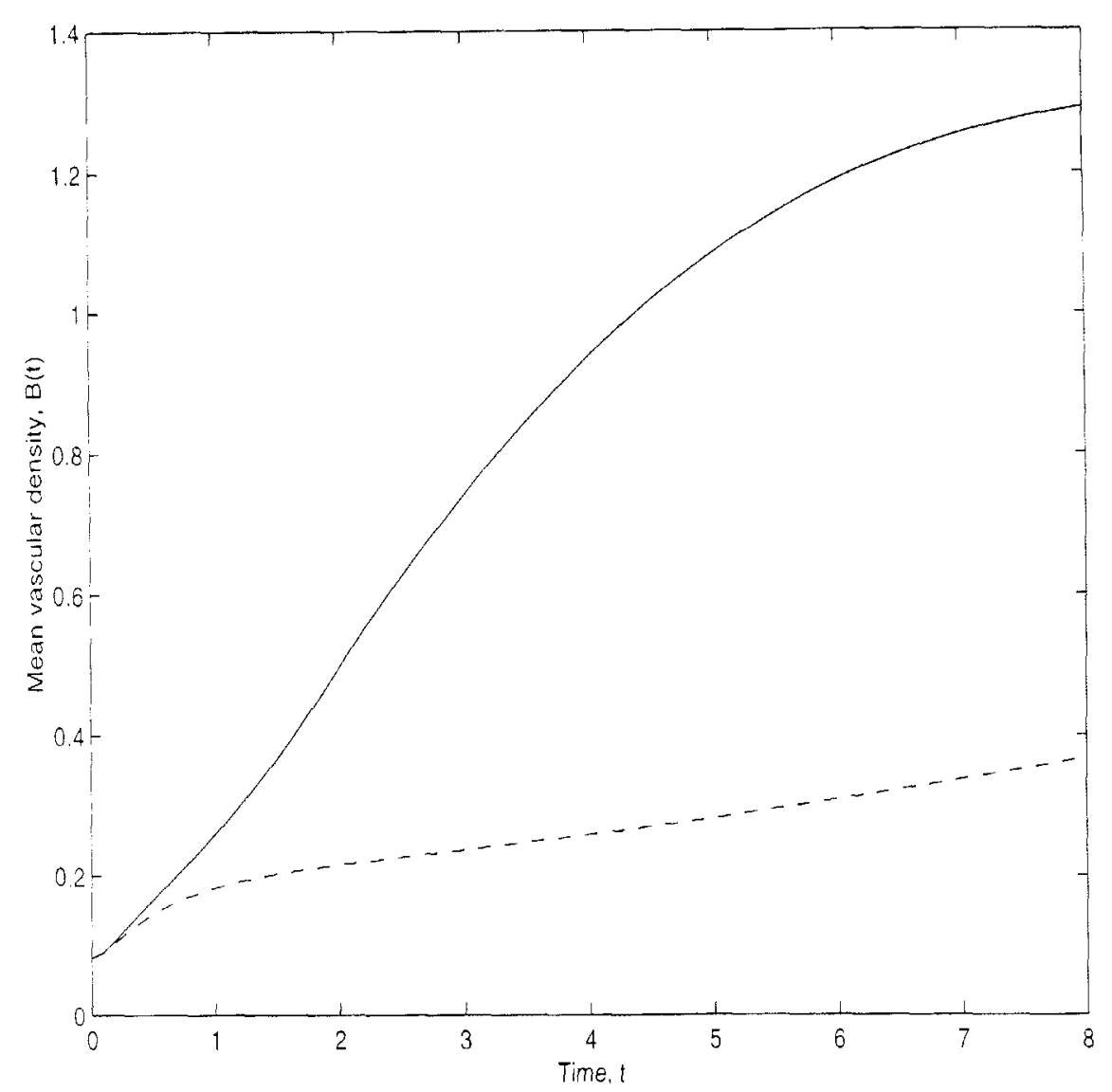

FIGURE 6 Numerical profiles showing how the mean vascular density $B(t)$ within the wound changes over time for the numerical simulations presented in Figures 3 and 4 . For the normal wound, $B(t) \rightarrow 1.3$ as $t \rightarrow \infty$. This corresponds to overhealing. Key: successful healing (solid line); failed healing (dashed line). Parameter values: as per Figures 3(a) and 4(a).

which is consistent with overhealing. By contrast $B(t)$ increases extremely slowly in the poorly healing wound. Comparing Figures 5 and 6 we suggest that monitoring the mean vascular density may prove to be a more discriminating indicator of the successful wound healing than estimates of the wound's surface area.

\subsection{Model Modification: Vessel Remodelling}

The simulations presented in Figures 3 and 6 suggest that a remodelling term should be included in Equation (3) in order to produce more realistic results, i.e. to ensure that the vessel density eventually returns to a normal level. Assuming that vascular remodelling is a continuous, logistic growth process, we now add a remodelling term to (3):

$$
\begin{aligned}
\left(\begin{array}{c}
\text { rate of increase } \\
\text { of vessel density }
\end{array}\right)= & \left(\begin{array}{c}
\text { rate of deposition } \\
\text { by tips }
\end{array}\right) \\
& +\left(\begin{array}{c}
\text { rate of } \\
\text { remodelling }
\end{array}\right)
\end{aligned}
$$

or, equivalently,

$$
\begin{aligned}
\frac{\partial b}{\partial t}= & \mu_{n} n \frac{\partial n}{\partial r}-\chi n \frac{\partial a}{\partial r} \\
& +\lambda_{6} b\left(b^{*}-b\right) .
\end{aligned}
$$

where $\lambda_{6}^{-1}$ is the remodelling timescale and, as in equations (5), $b^{*}$ is a characteristic vessel density in normal tissue. The (dimensionless) model equations, with (3) replaced by (8), were solved numerically and gave rise to profiles that were qualitatively similar to those presented in Figures 3 and 4. In order to assess the effect of the remodelling term, we plot, for different values of $\lambda_{6}$. the wound surface 
area $A(t)$ and the mean vessel density $B(t)$, and the results are presented in Figure 7. From the profiles of $A(t)$ we observe that the inclusion of a continuous remodelling term into our model reduces the time to wound closure for both normal and chronic wounds, the reduction in healing time being more pronounced for the chronic wound. From the profiles of $B(t)$, we observe that with remodelling the overhealing of the normal wound is no longer present: the vessel density increases monotonically to a level characteristic of normal tissue. Further, the remodelling term dramatically reduces the healing time of the slowly healing wound. Clearly this is physically unrealistic: the abnormally low density of capillary tips should prevent the wound from healing. In order to prevent such anomalies from occurring, the remodelling term used in Equation (8) should be modified. Therefore, we now assume that remodelling only commences when the new vessels meet at the centre of the wound. Thus the initial remodelling term of Equation (8) is superceded by the following expression:

$$
(\text { remodelling term })=\lambda_{6} H\left(t-T_{R}\right) b\left(b^{*}-b\right),
$$

where $T_{R}$ denotes the time at which remodelling commences and satisfies

$$
T_{R}=\min _{t}\{b(0, t)>0\}
$$

The effect that this delayed remodelling term has on angiogenesis is summarised in Figure 8 where we plot the mean vessel density $B(t)$. The top panel shows that until remodelling is activated, healing proceeds in exactly the same manner as in Figure 6, with the elevated vessel densities characteristic of newly healed wounds retained. However, once the vessels make contact at the wound centre the vessel density gradually decays to the vessel density characteristic of normal tissue. More importantly, we note, from the lower panel, that when the delayed remodelling term is applied to the poorly healing wound of Figure 6 the wound still fails to heal. In this case since the vessels have not penetrated to the wound centre the remodelling term is not activated. On the basis of the simulations presented in Figure 6 we suggest that the delayed term introduced above may describe the remodelling process that occurs during wound healing.

\subsection{Comparison between Experimental and Numerical Results}

In order to compare our mathematical model of angiogenesis with the experimental data, we assume, as above, that there is a correlation between the surface area of a wound (as measured experimentally) and the surface area of the avascular region of the tissue (as obtained from the numerical simulations). Before continuing we remark that a statistical fit of the model parameters to the experimental data was not performed. The paucity of data meant that the confidence limits would have been extremely large. Instead we sought model parameter values that gave good qualitative and quantitative agreement with the averaged data.

The two plots in Figure 9 show a comparison between the theoretical results of our model (from numerical simulations) and the actual clinical data. The top figure illustrates a normal wound healing while the lower figure illustrates a chronic wound. Theoretical data points are illustrated with a cross, while the actual data points are circles. Parameter values used in each case are given in the figure legend. The results presented in Figure 9 show that the model generates profiles of the wound surface area which are in good qualitative and quantitative agreement with the experimental data for both the normal and chronic wounds.

Guided by the differences in the model parameters that were needed to obtain numerical simulations that mimic normal and chronic wound healing we can suggest physical mechanisms that may be responsible for impaired wound healing. For example, our simulations suggest that a combination of reductions in (i) the sensitivity of the endothelial cells to the chemoattractant (measured by the chemotactic coefficient $\chi$ ) (ii) the proliferation rate of the endothelial cells (measured by the parameter $\lambda_{0}$ ) and (iii) chemoattractant production (measured by the parameter $\lambda_{3}$ ) may give rise to chronic wounds. 

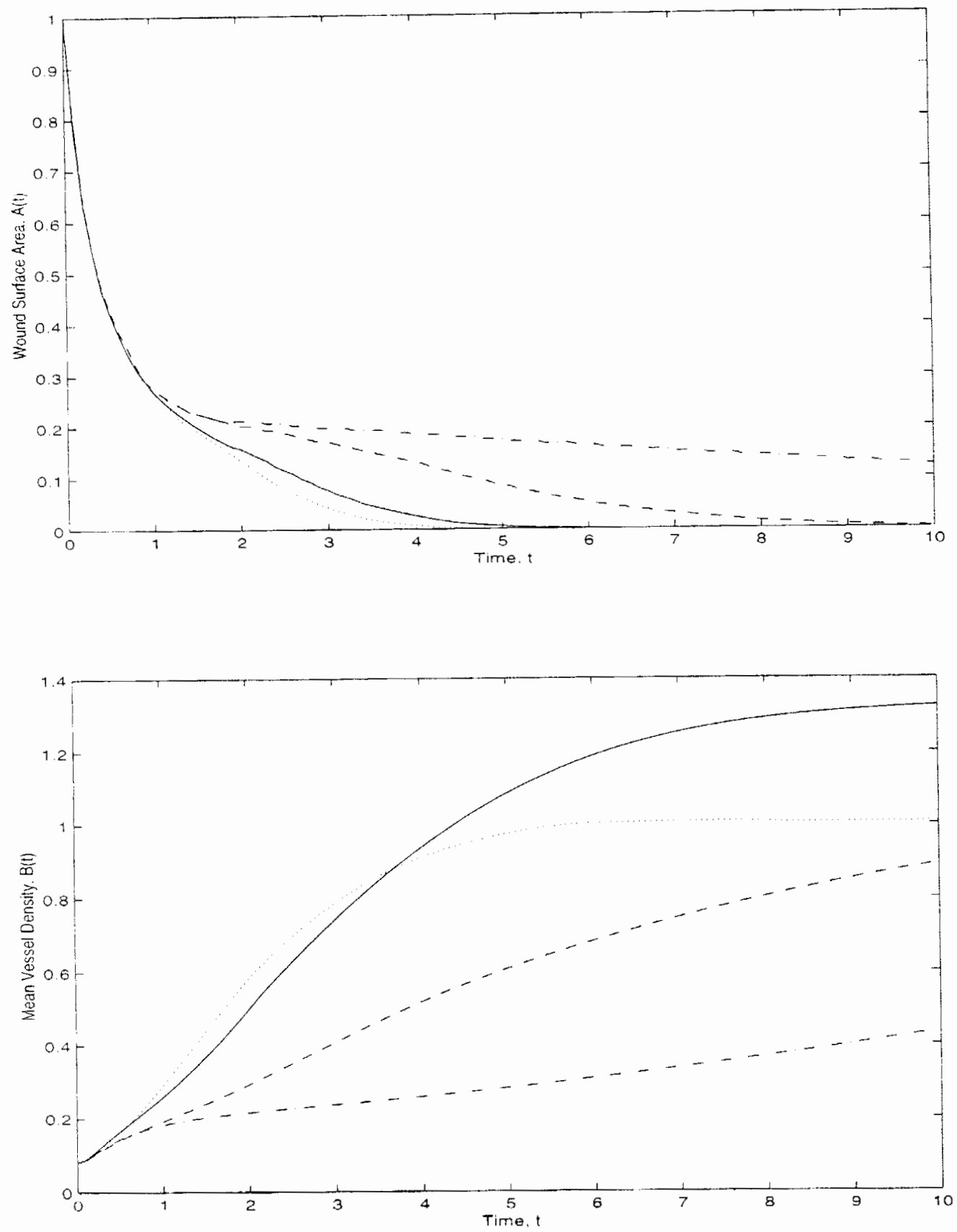

FIGURE 7 Numerical simulations showing the effect of introducing continuous vessel remodelling on the evolution of (a) the normalised wound surface area $A(t) / A(0)$ and (b) the mean vessel density $B(t)$. Key: normal healing, with no remodelling (solid line, $\lambda_{6}=0$ ); normal healing, with remodelling (dotted line, $\lambda_{6}=1.0$ ); failed healing, with no remodelling (dot-dash line, $\lambda_{6}=0$ ); failed healing, with remodelling (dashed line, $\lambda_{6}=1.0$ ). Parameter values: as per Figures 3 (a) and 4(a), with $\lambda_{6}=1.0$. 

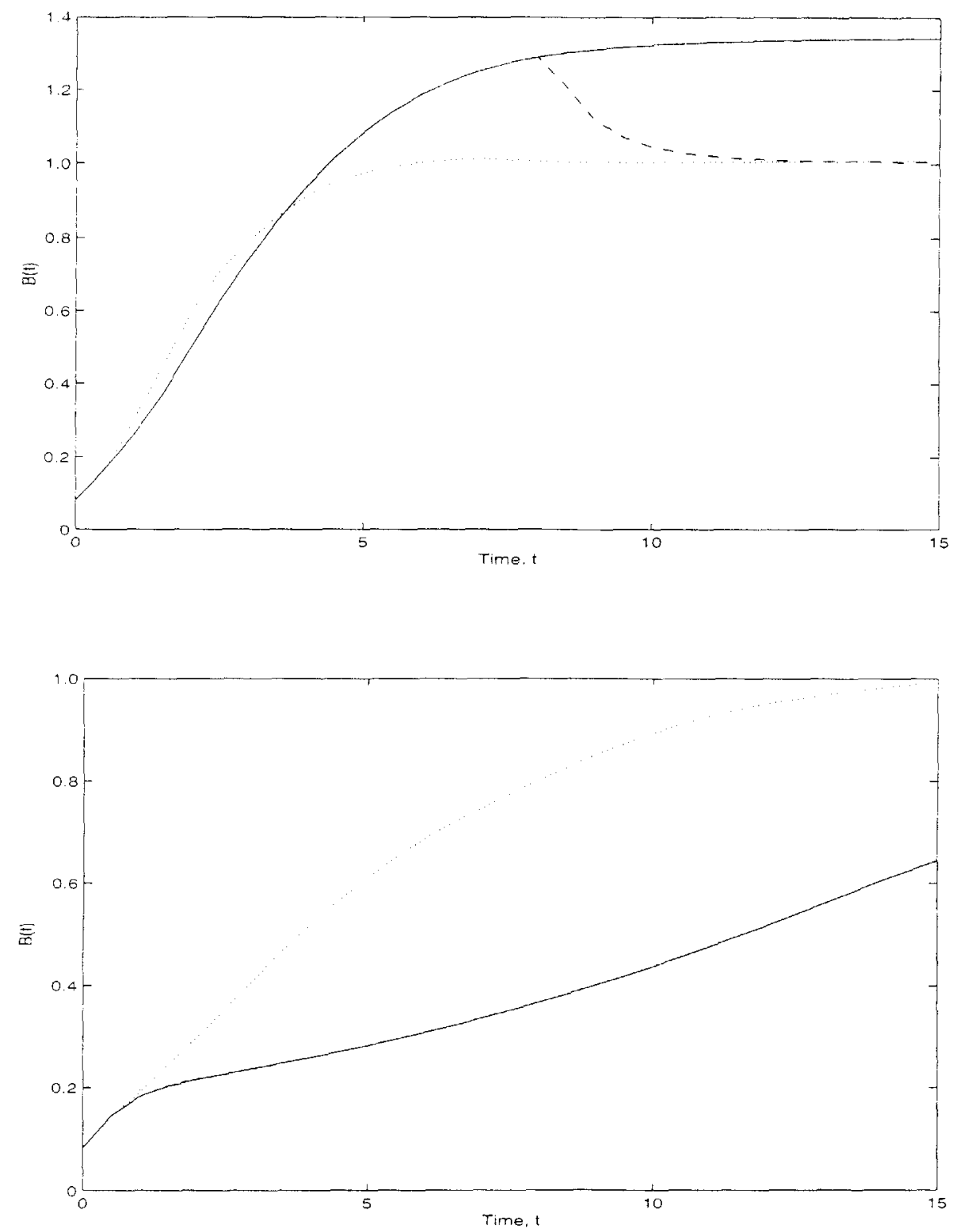

FIGURE 8 Numerical simulations showing the effect of using a time-delay in the vessel remodelling term on the evolution of the mean vessel density $B(t)$ : (a) normal healing; (b) chronic healing. Key to (a) and (b): no remodelling (solid line); continuous remodelling (dotted line); time-delay in remodelling (dashed line). Parameter values: as per Figure 7.

One of the advantages of mathematical models is that they enable us to determine the effect that changes in the relative importance of individual terms (or mechanisms) have on the healing process (such investigations are notoriously difficult or impossible to perform experimentally). Thus, having established that a combination of changes in $\chi, \lambda_{0}$ and $\lambda_{3}$ transforms a normally healing wound into a chronic wound, we can use the model to assess the relative importance of each parameter. For example, in Figure 10 we present numerical results that were obtained by varying the chemotaxis parameter $\chi$ over a physically realistic range while keeping all other parameters fixed. Estimates 
of $\chi$ from experimental data (c.f. Anderson and Chaplain. 1998, and references therein) show that its (nondimensional) value lies in the range 0.1 to 1.0. For each value of $\chi$ the time for the wound to heal was calculated and the results are plotted in Figure 10. From the results we can see clearly that as $\chi$ increases the time taken for healing to complete decreases. With more detailed parameter estimates it should be possible to interpret these results in a dimensional fashion. For example, by relating the dimensionless times to physical times (in weeks or days), it may be possible to identify a range of
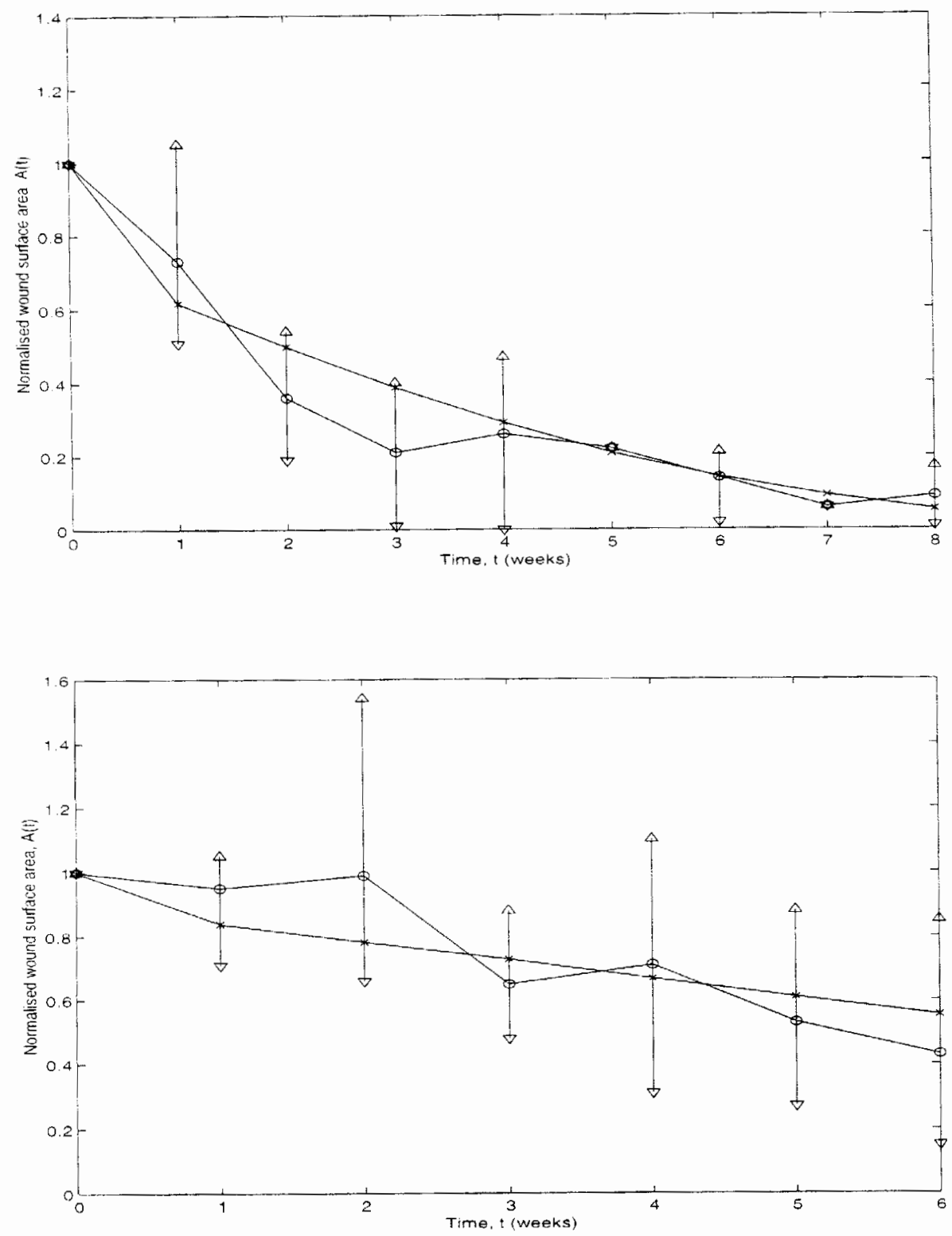

FIGURE 9 A comparison of theoretical results obtained from numerical simulations of our model and actual clinical data. Here we present numerical simulations that are in good qualitative agreement with the experimental data for normal and chronic healing Parameter values for simulations in case (a): $\mu_{n}=0.001, \chi=0.1, D=1, \lambda_{0}=50, \lambda_{1}=10, \lambda_{2}=10, \lambda_{3}=25=\lambda_{4}, \lambda_{5}=25, \lambda_{6}=5$ $R=1, R_{0}=0.95, k_{1}=2, N_{0}=1=b_{\text {norm }}=b_{\text {char }}, \Delta b=0.01$. Parameter values for simulations in case (b): $\chi=0.08, D=1, \lambda_{0}=5$, $\lambda_{1}=10 . \lambda_{2}=10, \lambda_{3}=5=\lambda_{4}, \lambda_{5}=25, \lambda_{6}=6, R=1, R_{0}=0.95, N_{0}=1=b_{\text {norm }}=b_{\text {char }}, \Delta b=0.01$. 


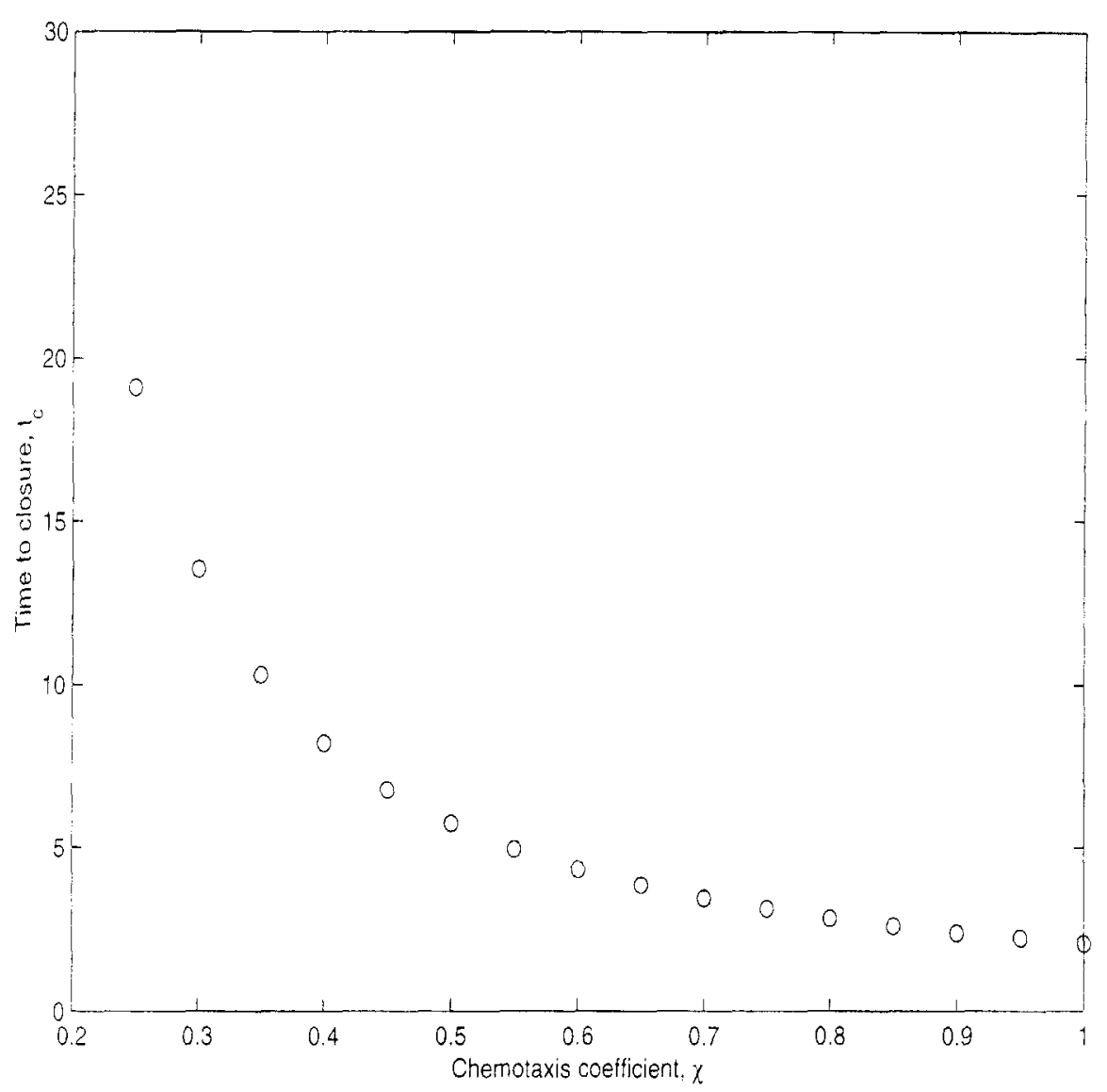

FIGURE 10 Sensitivity analysis of the parameter $\chi$. Illustration of the effect of changing the sensitivity of capillary tips to the chemoattractant (measured by the chemotactic coefficient $\chi$ ) on wound closure time, $t_{c}=\min _{i}\{b(0, t)>0\}$. As can be seen from the figure by increasing $\chi$ the time for the wound to heal decreases. Parameter values: $D=1, \lambda_{0}=50, \lambda_{1}=10, \lambda_{2}=10, \lambda_{3}=25=\lambda_{4}$, $\lambda_{5}=25, R=1, R_{0}=0.95, N_{0}=1=b_{\text {norm }}=b_{\text {char }} . \Delta b=0.01$.

values of the chemotaxis parameter $\chi \in\left(\chi_{k l}, \chi_{h i}\right)$ such that for $\chi>\chi_{h i}$ healing is successful whereas for $\chi<\chi_{l}$, the predicted healing time is so long that it is impractical. The same approach could also be used to estimate critical bounds for other model parameters including $\lambda_{0}, \lambda_{3}, \lambda_{6}$. Numerical studies performed by varying $\lambda_{0}$ and $\lambda_{3}$ gave rise to results similar to those presented in Figure 10, that is the time to healing decreases as $\lambda_{0}$ and $\lambda_{3}$ increase (results not included). The results of Figure 7 indicate that increasing the parameter $\lambda_{6}$ decreases the time for wound healing. Finally, we note that further numerical simulations carried out by varying the parameter $\lambda_{4}$ (not presented here), indicate that increasing $\lambda_{4}$ slows down the time for wound healing i.e. the wound takes longer to heal.

\section{CONCLUSIONS}

Wound healing is a complex process, involving many inter-connected events. To formulate a single mathematical model that includes all of the relevant processes would be a formidable task indeed. In this paper therefore we have modelled in a simple, but effective manner one key process of wound healing - angiogenesis - and related our theoretical results to actual clinical data. To achieve this we have focussed on several of the key events involved in angiogenesis during wound healing (namely capillary proliferation and migration and the production of angiogenic factors). Numerical simulations of the model equations were compared with independent clinical measurements of normal 
and chronic wounds, and good qualitative agreement obtained (see Figure 9) in both cases. By changing only a few parameter values in the model we were able to simulate both normal and chronic wound healing thus enabling us to identify the important mechanisms that may be responsible for impaired wound healing. These results were validated by comparing with the available experimental data.

Although the mathematical model is a simple one, it does capture many important features associated with angiogenesis in wound healing. Among these features are: the change in speed of capillary migration during the healing process i.e. the healing unit of the capillaries proceeds at an initially fast rate and then slows down: the model has been able to identify potential mechanisms responsible for failed or chronic healing and for vessel remodelling: the model has captured the elevated tip and vessel densities in newly healed wounds which is associated with the so-called "brush-border effect". Some refinement of the current model (such as the inclusion of a term modelling tip-vessel anastomosis, for example) may be considered in any future development and further numerical analysis of the model. A possible direction for future analytical work may involve using geometrical wave theory (Grindrod, 1996) to construct analytical model solutions that show explicitly how the different models parameters, such as $\chi, \lambda_{0}$ and $\lambda_{3}$, affect the speed with which healing progresses. However, since the capillary tips that are present in the healing unit are not necessarily all directly inter-connected, it may not be readily applicable to models of angiogenesis of the form presented in this paper.

As with all models, there are certain weaknesses in the approach we have adopted. Firstly, given that we have adopted a continuum model in one space dimension, the model is unable to capture the important process of anastomosis. One refinement of the current model as it stands could consider the inclusion of a term modelling tip-to-vessel anastomosis. However to capture this important process explicitly, a minimum requirement is that the analysis be carried out in (at least) 2 space dimensions and possibly also by adopting a discrete approach (cf. Chaplain and Anderson, 1997; Anderson and Chaplain, 1998). Indeed, it may be the case that the whole process of remodelling is triggered by tip-to-tip anastomosis, a fact that our model cannot account for. The model also ignores the important interaction between the capillaries and the extracellular matrix (although this is not quite as important in $1 \mathrm{D}$ as in 2D).

Nonetheless, the model has enabled us to make some observations and key predictions. It is clear that the migration of the capillaries is dominated by some form of "taxis"-either chemotaxis, in response to soluble cytokines such as angiogenic factors, or haptotaxis in response to response bound matrix proteins such as fibronectin (cf. Orme and Chaplain, 1997; Olsen et al., 1997; Anderson and Chaplain, 1998). The model has enabled us to speculate about possible mechanisms which are responsible (and, perhaps, important) for failed healing of wounds, namely, the response of capillaries to angiogenic factors, the production rate of angiogenic factors and the proliferation rate of the cells at the capillary tips. In particular, referring to the results presented in Figures 3 and 4 we predict that it may be possible to distinguish between different causes of impaired wound healing by observing the structure of the invading vascular front. Specifically, reasonable penetration of low density capillary tips may be attributed to a reduction in the tip proliferation rate whilst poor penetration and low capillary tip density may be due to a reduction in the sensitivity of the cells to chemotrattactants that are produced in the wound space.

In summary, our model has highlighted (a) the importance of vessel remodelling and this is certainly a development from previous models of angiogenesis in wound healing (Pettet etal., 1996a,b: Olsen et al., 1997) and (b) the importance of the actual functional form of the vessel remodelling term used in the model. Finally we note that the theoretical results of the model are such that to design experiments to test and verify or falsify the model predictions is certainly feasible (Orme and Chaplain, 1997). In particular, from the results of our model simulations, we assert that measurement 
of the mean vascular density may prove to be a more discriminating indicator of successful wound healing than simple estimates of the wound's surface area.

\section{APPENDIX}

In this appendix we show how the model equations of Section 3 were cast in dimensionless form prior to the construction of numerical solutions in Section 4. We introduce dimensionless variables

$$
\begin{aligned}
& \nu=n / N, \quad \alpha=a / A, \quad \beta=b / B, \\
& \rho=r / R \quad \text { and } \quad \tau=t / T,
\end{aligned}
$$

where $R$ denotes the size of the tissue region of interest and the constants $N, A, B$ and $T$ denote typical values of the corresponding dependent and independent variables which are defined in terms of system parameters in the following way:

$$
\begin{aligned}
& N=B / R, \quad A=\lambda_{3} / \lambda_{4}, \\
& B=b_{\text {nom }} \quad \text { and } \quad T=R^{2} / D .
\end{aligned}
$$

Rewriting Equations (1-7) in terms of the dimensionless variables we obtain the following system of equations which were used to compute the numerical simulations:

$$
\begin{aligned}
\frac{\partial \nu}{\partial \tau}= & \frac{\bar{\mu}_{n}}{\rho} \frac{\partial}{\partial \rho}\left(\rho \nu \frac{\partial \nu}{\partial \rho}\right)-\frac{\bar{\chi}}{\rho} \frac{\partial}{\partial \rho}\left(\rho \nu \frac{\partial \alpha}{\partial \rho}\right) \\
& +\bar{\lambda}_{0} \alpha \beta-\bar{\lambda}_{1} \nu-\bar{\lambda}_{2} \nu^{2}, \\
\frac{\partial \alpha}{\partial \tau}= & \frac{1}{\rho} \frac{\partial}{\partial \rho}\left(\rho \frac{\partial \alpha}{\partial \rho}\right) \\
& +\frac{\bar{\lambda}_{3}}{2}\left\{\tanh \left(\frac{\beta_{\text {char }}-\beta}{\Delta \beta}\right)+1\right\} \\
& -\bar{\lambda}_{3} \alpha-\bar{\lambda}_{5} \alpha \beta . \\
\frac{\partial \beta}{\partial \tau}= & \bar{\mu}_{n} \nu \frac{\partial \nu}{\partial \rho}-\bar{\chi} \nu \frac{\partial \alpha}{\partial \rho}
\end{aligned}
$$

with

$$
\begin{aligned}
& \nu(\rho, 0)=0=\alpha(\rho, 0) \\
& \beta(\rho, 0)= \begin{cases}0 & \rho \in\left(0, \bar{R}_{0}\right), \\
1 & \rho \in\left(\bar{R}_{0}, 1\right)\end{cases}
\end{aligned}
$$

$$
\begin{aligned}
\frac{\partial \nu}{\partial \rho}(0, \tau)= & 0=\frac{\partial \alpha}{\partial \rho}(0, \tau), \\
\nu(1, \tau)= & \bar{N}_{0} e^{-\bar{k}_{1} \tau}, \\
& D \frac{\partial \alpha}{\partial \rho}(1, \tau)=-\bar{k}_{2} \alpha(1, \tau) \beta(1, \tau),
\end{aligned}
$$

where

$$
\begin{aligned}
\bar{\mu}_{n} & =\frac{\mu_{n} N T}{R^{2}}, \quad \bar{\chi}=\frac{\chi A T}{R^{2}}, \\
\beta_{\text {char }} & =\frac{b_{\text {char }}}{B}, \quad \Delta \beta=\frac{\Delta b}{B}, \\
\bar{\lambda}_{0} & =\frac{\lambda_{0} A B T}{N}=\frac{\lambda_{0} \lambda_{3} R}{\lambda_{4}}, \quad \bar{\lambda}_{1}=\lambda_{1} T, \\
\bar{\lambda}_{2} & =\lambda_{2} N T, \quad \bar{\lambda}_{3}=\frac{\lambda_{3} T}{A}, \quad \bar{\lambda}_{5}=\lambda_{5} B T, \\
\bar{N}_{0} & =\frac{N_{0}}{N}, \quad \bar{k}_{1}=k_{1} T, \quad \bar{k}_{2}=k_{2} B R .
\end{aligned}
$$

\section{References}

Anderson, A. R. A. and Chaplain, M. A. J. (1998). Continuous and discrete mathematical models of tumour-induced angiogenesis. Bull. Math. Biol., 60, 857-899.

Arnold, F. and West, D. C. (1991). Angiogenesis in wound healing. Pharmac. Ther., 52, 407-422.

Balding, D. and McElwain, D. L. S. (1985). A mathematical model of tumour-induced capillary growth. J. theor. Biol., 114, 53-73.

Bennet, N. T. and Schultz, G. S. (1993). Growth factors and wound healing: Part II role in normal and chronic wound healing. The American Ioumal of Surgery, 166, 74-81.

Byrne, H. M. and Chaplain, M. A. J. (1995). Mathematical models for tumour angiogenesis: Numerical simulations and nonlinear wave solutions. Bull. Math. Biol., 57, 461-486.

Canfield, A. E. and Schor, A. M. (1995). Evidence that tenascin and thrombospondin- 1 modulate sprouting of endothelial cells. J. Cell Sci., 108, 797-809.

Chaplain, M. A. J. and Byrne, H. M. (1996). The mathematical modelling of wound healing and tumour growth: two sides of the same coin. Wounds, 8, 42-48,

Chaplain, M. A. J. and Anderson, A. R. A. (1997). Mathematical modelling, simulation and prediction of tumour-induced angiogenesis. Invasion and Metastasis, 16, 222-234.

Clark, R. A. F. (1988). Overview and general considerations of wound repair. In: The Molecular and Cellular Biology of Wound Repair, (ed. Clark, R. A. F. \& Henson, P. M.), pp. 3-33. New York: Plenum Press

Claesson-Welsh, L., Welsh, M., Ito, N., AnandApte, B. Soker, S., Zetter, B., O'Reilly, M. and Folkman, J. (1998). Angiostatin induces endothelial cell apoptosis and activation of focal adhesion kinase independently of the integrin-binding motif RGD. Proc. Nat. Acad. Sci. USA, 95, 5579-5583. 
Cliff, W. J. (1963). Observations on healing tissue: A combined light and electron microscopic investigation. Trans. Roy. Soc. London, B246, 305-325.

Cliff. W. J. (1965). Kinetics of wound healing in rabbit car chambers, a time lapse cinemicroscopic study. Quart. Jl. Exp. Physial. Cog. Med. Sci., 50, 79-89.

Dale, P. W., Maini, P. K. and Sherratt, J. A. (1994). Mathematical modelling of corneal epithelial wound healing. Math. Biosci, 124, $127-147$.

Dale, P. W.. Sherratt, J. A. and Sherratt, P. K. (1996). A mathematical model for collagen fibre formation during fetal and adult dermal wound-healing. Proc. Roy. Soc. Lond. Ser. B, $\mathbf{2 6 3}$, $653-660$.

Dale, P. W., Sherratt, J. A. and Maini, P. K. (1997). Role of fibroblast migration in collagen fibre formation during fetal and adult dermal wound healing. Bull. Math. Biol., 59 $1077-1100$.

Dallon, J. C. and Sherratt, J. A. (1998). A mathematical model for fibroblast and collagen orientation. Bull. Math. Biol., 60, $101-129$.

Davidson, J. (1995). Growth-factors in wound healing. Wounds, 7. A53-A64.

Davidson, J. M. and Broadley, K. N. (1991). Manipulation of the wound-healing process with basic fibroblast growth factor. Ann. New York Acad. Sci., 638, 306-315.

Folkman, J. (1976). The vascularisation of tumors. Sci. Am., 234, $58-73$.

Folkman. J. and Brem, H. (1992). Angiogenesis and inflammation. In: Inflammation: Basic Principles and Clinical Correlates, Second Edition. (eds. Gallin, J. I., Goldstein, I. M. \& Snyderman, R.). New York:Raven Press.

Gimbrone, M. A., Cotran, R. S., Leapman, S. B. and Folkman, J. (1974). Tumor growth and neovascularisation: An experimental model using the rabbit cornea. J. Natn. Cancer Inst., 52, 413-427.

Graham, C. H. and Lala, P. K. (1992). Mechanisms of placental invasion of the uterus and their control. Biochem. Cell Biol. 70, $867-874$

Grindrod, P. (1996). The theory and applications of reactiondiffusion equations patterns and waves. O.U.P.

Hamilton, J. A. (1983). Hyothesis - in vitro evidence for the invasion and tumour-like properties of rheumatoid pannus. $J$ Rheum., 10, 845-851.

Knighton, D. R., Silver, I. A. and Hunt, T. K. (1981). Regulation of wound-healing angiogenesis-effect of oxygen gradients and inspired oxygen concentration. Surgery, 90, 262-270.

Knighton, D. R., Hunt, T. K., Scheunstuhl, H. and Halliday, B. J. (1983). Oxygen tension regulates the expression of angiogenesis factor by macrophages. Science, 221. $1283-1285$.

Knighton, D. R. and Fiegel, V. D. (1989). Macrophage-derived growth factors in wound healing: regulation of growth factor production by the oxygen microenvironment. Am. Rev. Respir. Dis., 140, 1108-1111.

Li, J., Brown, L. F., Hibberd, M. G., Grossman, J. D. and Simons, M. (1996). VEGF, FLK-1 and FLT-1 expression in a rat myocardial-infarction model of angiogenesis. Am. J. Physiol. Heart and Circulatory Physiol., 39. H1803-HI8I 1

Miller, J. W., Adams, A. P. and Aiello, L. P. (1997). Vascular endothelial growth factor in ocular neovascularisation and proliferative diabetic retinopathy. Diabetes-Metabolism Rev. 13, 37-50.

Muthukkaruppan, V. R., Kubai, L. and Auerbach, R. (1982). Tumor-induced neovascularisation in the mouse eye. J. Natn. Cancer Inst., 69, 699-705.
Myers, D. B. and Broom, N. D. (1982). Morphological and biomechanical studies of rheumatoid pannus and cartilage. $J$. Rheum., 9, 502-513.

Niinikoski, J., Heughan, C. and Hunt, T. K. (1971). Oxygen and carbon dioxide tensions in experimental wounds. Surg. Gyn. Obst. 133, 1003-1007.

O'Kane, S. and Ferguson, M. W. J. (1997). Transforming growth factor betas and wound healing. Intl. J. Biochent. Cell Biol., 29, 63-78.

Olsen, L. Sherratt, J. A. and Maini. P. K. (1995). A mechanochemical model for adult dermal wound contraction and the permanence of the contracted tissue displacement profile. $J$. theor. Biol. 177, 113-128.

Olsen, L., Sherratt, J. A. and Maini, P. K. (1996). A mathematical model for fibro-proliferative wound-healing disorders. Bull. Math. Biol, 58, 787-808.

Olsen, L., Sherratt, J. A., Maini, P. K. and Arnold, F. (1997a). A mathematical model for the capillary endothelial cellextracellular matrix interactions in wound-healing angiogenesis. IMA J. Math. Appl. Med. Biol., 14, 261-281.

Olsen, L Maini, P and Sherratt, J. A (1997b). A mechanochemical model for normal and abnormal dermal wound repair. Nonlinear Analysis-Theory Methods and Applications. 30, 3333-3338.

Olsen, L., Maini, P. K. and Sherratt, J. A. (1998). Spatially varying equilibria of mechanical models: Application to dermal wound contraction. Math. Biosci. 147, 113-129.

Orme, M. E. and Chaplain, M. A. J. (1997). Two-dimensional models of tumour angiogenesis and anti-angiogenesis strategies, IMA J. Math. Appl. Med. Biol, 14, 189-205

Paget, C. J., Wolk, S. W., Whitehouse, W. M., Erlandson, E. E., Landman, R. M., Schaldenbrand, J. and Fry, W. J. (1997). Incidence and pathophysiologic significance of infected carotid arter plaque. Ann. Vasc. Surgery, 11, 129-132.

Pacues, M., Massin, P. and Gaudric, A. (1997). Growth factors and diabetic retinopathy. Diabetes Metab., 23, 125-130

Paweletz, N. and Knierim, M. (1989). Tumor-related angiogenesis. Crit. Rev. Oncol. Hematol, 9, 197-242.

Pettet, G. J., Byrne, H. M., McElwain, D. L. S. and Norbury, J. (1996a). A model of wound-healing angiogenesis in soft tissue. Math. Biosci., 136, 35-63.

Pettet, G., Chaplain, M. A. J., McElwain, D. L. S. and Byrne, H. M. (1996b). On the role of angiogenesis in wound healing. Proc. Roy. Soc. Ser. B, 263, $1487-1493$.

Phillips, G. D., Stone, A. M., Schultz, J. C., Jones, B. D., Goodkin, M. L., Lisowski, M. J., White-head, R. A., Fiegel, V. D. and Knighton, D. R. (1997). Do growth factors stimulate angiogenesis? A comparison of putative angiogenesis factors. Wounds, 9, $1-14$

Polverini, P. J., Cotran, R. S., Gimbrone, Jr. M. A. and Unanue, E. R. (1977). Activated macrophages induce vascular proliferation. Nature, 269, 804-806.

Schoefl, G. I. (1963). Studies on inflammation III. Growing capillaries: Their structure and permeability. Virchows Arch. Pathol. Anat., 337, 97-141.

Shah, M., Foreman, D. M. and Ferguson, M. W. J. (1995). Neutralisation of TGF- $\beta(1)$ and TGF- $\beta(2)$ or exogenous addition of TGF- $\beta(3)$ to cutaneous rat wounds reduces scarring. $J$. Cell Sci. 108, 985- 1002 .

Sherratt, J. A. and Murray, J. D. (1990). Models of epidermal wound healing. Proc. $R$. Soc. Lond. B, 241, 29-36.

Shinohara, K., Shinohara, T., Mochizuki, N., Mochizuki, Y. Sawa, H., Kohya, T., Fujita, M., Fujioka, Y., Kitabatake, A and Nagashima, K., (1996). Expression of vascular endothelial growth factor in human myocardial infarction. Heart and Vessels, 11, 113-122 
Sholley, M. M., Gimbrone, M. A. and Cotran, R. S. (1977). Cellular migration and replication in endothelial regeneration. Lab. Invest., 36, 18.

Sholley, M. M., Ferguson, G. P., Seibel, H. R., Montour, J. L. and Wilson, J. D. (1984). Mechanisms of neovascularisation. Vascular sprouting can occur without proliferation of endothelial cells. Lab. Invest., 51, 624-634.

Stokes, C. L., Rupnick, M. A., Williams, S. A. and Lauffenburger, D. A. (1990). Chemotaxis of human microvessel endothelial cells in response to acidic fibroblast growth factor. Lab. Invest., 63, 657-668.

Stokes, C. L. and Lauffenburger. D. A. (1991). Analysis of the roles of microvessel endothelial cell random motility and chemotaxis in angiogenesis. J, theor. Biol., 152, 377-403.
Terranova, V. P., Diflorio, R., Lyall, R. M., Hic, S., Friescl, R. and Maciag, T. (1985). Human endothelial cells are chemotactic to endothelial cell growth factor and heparin. $J$. Cell Biol., 101, 2330-2334.

Ungari, S., Katari, R. S., Alessandri, G. and Gullino, P. M. (1985). Cooperation between fibronectin and heparin in the mobilization of capillary endothelium. Invas. Metast, 5. $193-205$.

Warren, B. A. (1966). The growth of the blood supply to melanoma transplants in the hamster check pouch. Lab. Invest., $15,464-473$.

Wilcken, D. E. L. and Wilcken, B. (1997). The natural history of vascular disease in homocystinuria and the effects of treatment. J. Inherited Metabol. Disease, 20, 295-300. 


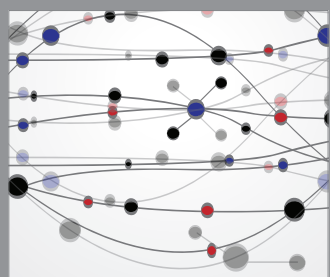

The Scientific World Journal
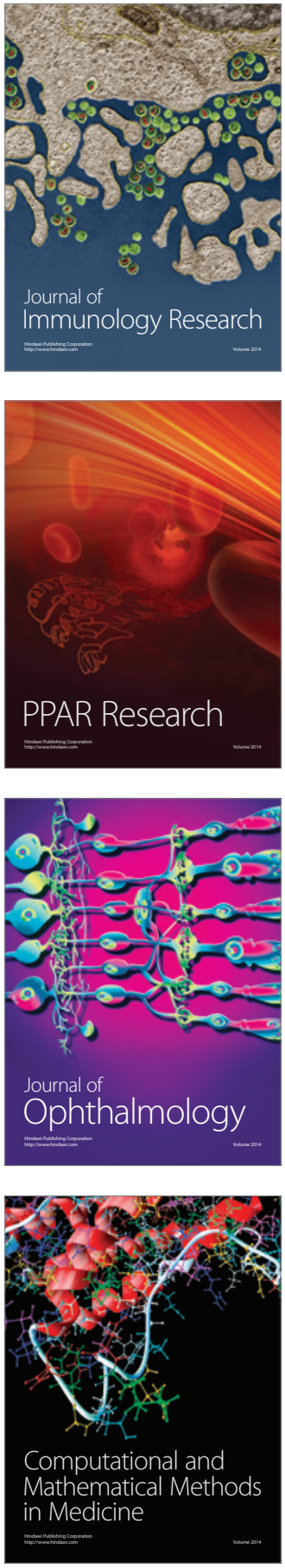

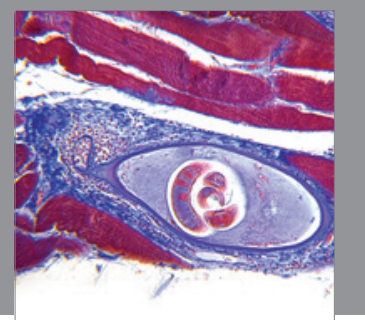

Gastroenterology

Research and Practice
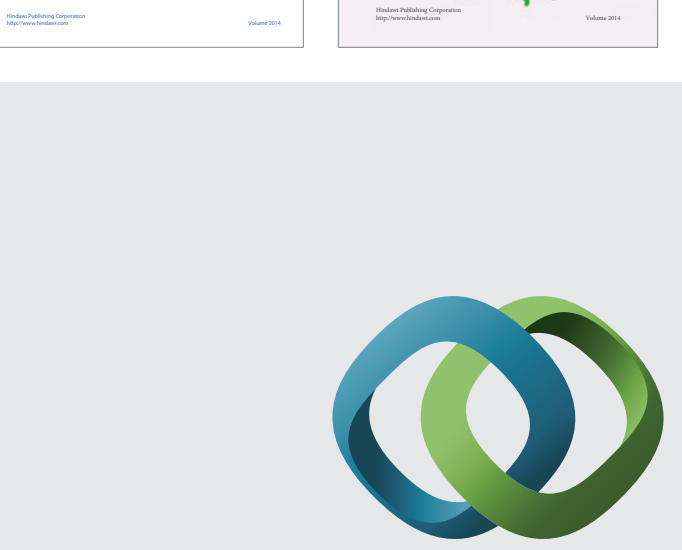

\section{Hindawi}

Submit your manuscripts at

http://www.hindawi.com
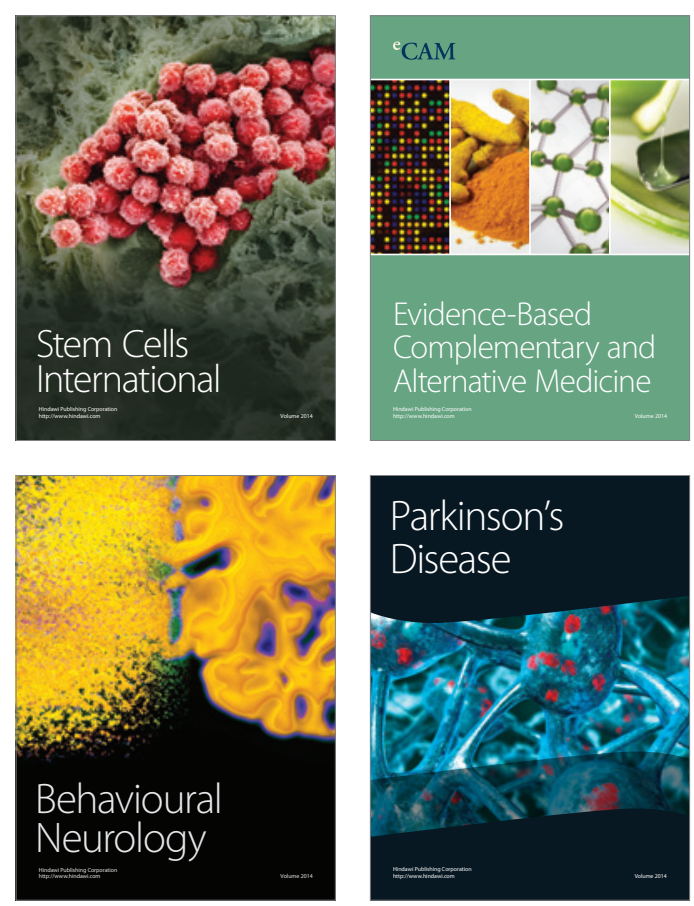

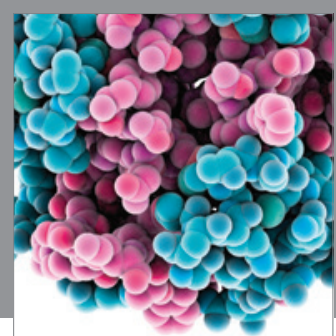

Journal of
Diabetes Research

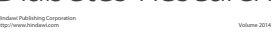

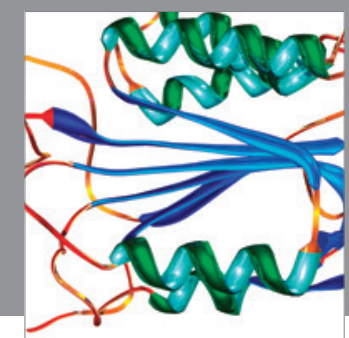

Disease Markers
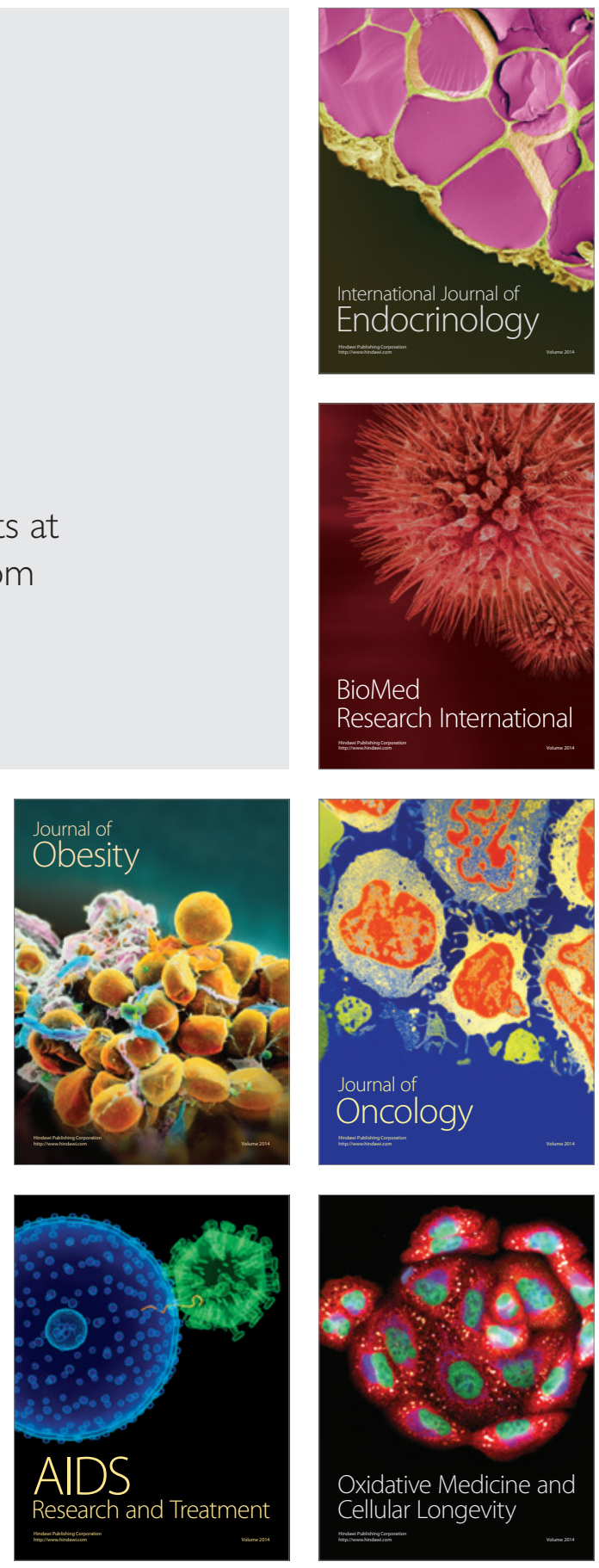